\title{
The Outer Halo of the Milky Way as Probed by RR Lyr Variables from the Palomar Transient Facility*
}

\author{
Judith G. Cohen ${ }^{1}$ (1) , Branimir Sesar ${ }^{1,2}$ (1) , Sophianna Bahnolzer ${ }^{3}$, Kevin $\mathrm{He}^{1}$, Shrinivas R. Kulkarni ${ }^{1}$ (i), Thomas A. Prince ${ }^{1}$ (D), \\ Eric Bellm ${ }^{1,4}$ (1), and Russ R. Laher ${ }^{5}$ \\ ${ }^{1}$ Division of Physics, Mathematics and Astronomy, California Institute of Technology, Pasadena, CA 91125, USA; jlc@astro.caltech.edu, khe@caltech.edu, \\ srk@astro.caltech.edu, prince@srl.caltech.edu, ebellm@astro.caltech.edu \\ ${ }^{2}$ Max Planck Institute for Astronomy, Konigstuhl 17, D-69117, Heidelberg, Germany; bsesar@mpia.de \\ 3535 Pontius Ave. N., Apt. 507, Seattle, WA 98109, USA; sophianna.banholzer@gmail.com \\ ${ }^{4}$ Box 351580, University of Washington, Seattle, WA 98195, USA; ecbellm@uw.edu \\ ${ }^{5}$ Infrared Processing and Analysis Center, California Institute of Technology, Pasadena, CA 91125, USA \\ Received 2017 April 26; revised 2017 September 26; accepted 2017 September 28; published 2017 November 9
}

\begin{abstract}
RR Lyrae stars are ideal massless tracers that can be used to study the total mass and dark matter content of the outer halo of the Milky Way (MW). This is because they are easy to find in the light-curve databases of large stellar surveys and their distances can be determined with only knowledge of the light curve. We present here a sample of $112 \mathrm{RR}$ Lyr stars beyond $50 \mathrm{kpc}$ in the outer halo of the MW, excluding the Sgr streams, for which we have obtained moderate-resolution spectra with Deimos on the Keck II Telescope. Four of these have distances exceeding $100 \mathrm{kpc}$. These were selected from a much larger set of 447 candidate RR Lyr stars that were datamined using machine-learning techniques applied to the light curves of variable stars in the Palomar Transient Facility database. The observed radial velocities taken at the phase of the variable corresponding to the time of observation were converted to systemic radial velocities in the Galactic standard of rest. From our sample of $112 \mathrm{RR}$ Lyr stars we determine the radial velocity dispersion in the outer halo of the MW to be $\sim 90 \mathrm{~km} \mathrm{~s}^{-1}$ at $50 \mathrm{kpc}$, falling to about $65 \mathrm{~km} \mathrm{~s}^{-1}$ near $100 \mathrm{kpc}$ once a small number of major outliers are removed. With reasonable estimates of the completeness of our sample of 447 candidates and assuming a spherical halo, we find that the stellar density in the outer halo declines as $r^{-4}$.
\end{abstract}

Key words: Galaxy: halo - Galaxy: kinematics and dynamics - stars: variables: RR Lyrae

Supporting material: machine-readable tables

\section{Introduction}

We present initial results of a study of the outer halo of the Milky Way (MW) using a large sample of RR Lyrae type $a b$ (denoted as RRab) variables. RR Lyr are old low-mass pulsating stars with distinctive light curves, amplitudes at $R$ of $\sim 0.5-1 \mathrm{mag}$, and periods of $\sim 0.4-0.8$ days, which are unchanged on a timescale of years and in most cases decades or longer. These characteristics make them fairly easy to distinguish in a wide-field, multiepoch optical imaging survey if the survey cadence is suitable. Their most desirable characteristic is that they are standard candles. Accurate luminosities, which have only a small dependence on metallicity and period (see the discussion in Section 4), can be inferred directly from the light curves, and these stars, with $M_{V} \sim+0.6 \mathrm{mag}$, are fairly luminous and hence can be detected at large distances.

There is a long history reaching back more than $30 \mathrm{yr}$ of efforts to calibrate the RR Lyr period-luminosity-metallicity relation, many of which use the Baade-Wesselink (Baade 1926; Wesselink 1969) infrared surface brightness technique to establish an accurate distance scale. Their subsequent use as distance indicators within the MW halo, primarily for globular clusters and distant halo stars, also has a long history; see, e.g., Longmore et al. (1986), Cohen (1992), and many other early

\footnotetext{
* Based in part on observations obtained at the W. M. Keck Observatory, which is operated jointly by the California Institute of Technology, the University of California, and the National Aeronautics and Space Administration.
}

efforts. Early calibration efforts (see, e.g., Longmore et al. 1990) demonstrated the advantages of using IR photometry rather than optical photometry, specifically lower amplitude of variation, meaning that fewer epochs are required to determine a mean magnitude and hence a luminosity. Now with the Spitzer IRAC camera (Fazio et al. 2004; Werner et al. 2004) and the WISE all-sky survey (Wright et al. 2010), highly accurate photometry on a stable space-based platform enables even more precise distances for RR Lyr, with recent periodluminosity calibrations for the WISE bandpasses carried out by Madore et al. (2013) and Klein et al. (2014). Furthermore, the Hubble Space Telescope Fine Guidance Sensor cameras were used by Benedict et al. (2011) to determine trigonometric parallaxes to several of the nearest field RR Lyr, in principle providing a fundamental calibration for all these efforts. Sesar et al. (2017a) used the Tycho-Gaia Astrometric Solution (Michalik et al. 2015) parallaxes of nearby RR Lyr from the Gaia Data Release 1 (Brown et al. 2016) to verify existing period-luminosity-metallicity relationships of previous studies, illustrating the potential for very high accuracy distances for RR Lyr stars with future Gaia releases.

Our survey for RR Lyr is focused on fundamental mode pulsators, i.e., RR Lyr type $a b$. Type $c$ RR Lyr stars, which are first-overtone pulsators, compose roughly $23 \%$ of the total RR Lyr population (Soszynski et al. 2016). They are systematically less luminous than RRab by about 0.25 mag and have shorter periods (Braga et al. 2015). RR Lyr type $c$ can be distinguished from fundamental mode pulsators by their smoother, more 
sinusoidal light curves, but this makes their separation from contact binary systems more challenging.

We are now in an era of large digital imaging surveys, including the Sloan Digital Sky Survey (SDSS; York et al. 2000), the Palomar Transient Facility (PTF; Law et al. 2009; Rau et al. 2009), and its successors, the Catalina Real-Time Transient Surveys (CRTS; Mahabel et al. 2011; P.I. S. G. Djorgovski) and the Pan-STARRS survey (Hodapp et al. 2004; Tonry et al. 2012), with the Large Synoptic Survey Telescope (LSST) coming in the next decade. Gaia recently had its first data release as well (Brown et al. 2016), with more to follow in due course. These surveys, with their huge databases, can, depending on their cadences and limiting magnitudes, be used to identify ever-larger samples of ever more distant RR Lyr, continuing and expanding on much earlier efforts (see, e.g., Wetterer \& McGraw 1996). Such samples enable studies of the outer halo of the MW, as well as of streams and substructures therein. RR Lyr are particularly useful for isolating halo substructures, as they stand out through their variability and blue color against the numerous foreground Galactic disk and inner halo stars; Sesar et al. (2012, 2013a) have utilized the PTF samples for this purpose.

Our survey for RR Lyr in the outer halo of the Galaxy carried out with the PTF begins at a heliocentric distance of $50 \mathrm{kpc}$ and reaches out to distances of $\sim 110 \mathrm{kpc}$. Previous surveys of halo RR Lyr stars include Vivas et al. (2001), Keller et al. (2008), Miceli et al. (2008), Sesar et al. (2011, 2013b), and Drake et al. (2014), among others. Our survey presents a significant improvement over anything previously published in sample size and in precision of distances in the $50-100 \mathrm{kpc}$ range. We present in this paper the radial velocity data obtained to date for these distant RR Lyr through moderate-resolution spectroscopy at the Keck Observatory, as well as a preliminary halo density distribution derived from our full RR Lyr sample.

An overview of the PTF is given in Section 2. In the following sections we briefly review how we derived our RR Lyr sample and then describe how we calculate distances from the light-curve parameters. We then discuss our spectroscopic follow-up campaign at the Keck Observatory to measure radial velocities, and we present $v_{r}$ for 112 RR Lyr in the outer halo of the MW with heliocentric distances ranging from 50 to $109 \mathrm{kpc}$, and with a median distance of $73 \mathrm{kpc}$, which we subsequently use to derive the velocity dispersion in the outer halo of the MW. Next, we give a description of our preliminary halo density distribution derived from our full sample of 447 RR Lyr candidates. This is followed by a comparison of our results with the results of other recent studies of the outer halo of the MW, and then a summary.

\section{Overview of the PTF}

The PTF (Law et al. 2009; Rau et al. 2009; P.I. S. R. Kulkarni of Caltech) is a wide-area, two-band ( $g$ and $R$ filters), deep $(R \sim 20.6$ single epoch, $\sim 23 \mathrm{mag}$ co-added) survey aimed at systematic exploration of the optical transient sky. The PTF ran for $3 \mathrm{yr}$, ending 2012 December 31, and then transitioned to the intermediate-PTF (iPTF), with the same goals and facilities but with a slightly different consortium membership. The project uses the CFH12k mosaic camera, with a field of view of $7.26 \mathrm{deg}^{2}$ and a plate scale of $1^{\prime \prime} \mathrm{pixel}^{-1}$, mounted on the Palomar Observatory 48-inch Samuel Oschin Schmidt Telescope (Rahmer et al. 2008). The camera consists of two rows of six $2 k \times 4 k$ CCDs, one of which is not active.
By the end of 2014 September, $\sim 12,000 \mathrm{deg}^{2}$ of sky had been observed by the iPTF in the Mould $R$ filter ${ }^{6}$ and $\sim 2300 \mathrm{deg}^{2}$ in the SDSS $g^{\prime}$ filter at least 30 times each. Observations are carried out with several cadences to support various major projects, ranging from searches for comets and asteroids to discovery and monitoring of distant supernovae (SNe). For most of a lunation, the observations are performed in a broadband $R$ filter. The SDSS $g^{\prime}$ filter is used during the darkest nights. Under typical seeing conditions (1"! 1 at the P48 Schmidt), the camera achieves an FWHM intensity of $\sim 2$ !" 0 and $5 \sigma$ limiting $\mathrm{AB}$ magnitudes of 20.6 in median seeing.

All PTF data taken by the Palomar Observatory 48-inch telescope are automatically routed to two pipelines: a real-time transient detection pipeline optimized for rapid detection of interesting objects, mostly $\mathrm{SNe}$, and hosted by the Lawrence Berkeley National Lab, and a longer-term archival pipeline optimized for high-precision photometry and hosted by the Infrared Processing and Analysis Center (IPAC). The IPAC pipeline performs final image reduction, source extraction, and photometric and astrometric calibration (Grillmair et al. 2010; Ofek et al. 2012; Laher et al. 2014). The photometric uncertainty provided by this pipeline is smaller than $\sim 0.01 \mathrm{mag}$ for $R<16$ sources and increases to $0.2 \mathrm{mag}$ at $R=20.6$. The algorithm used for photometric calibration is based on that of Honeycutt (1992) as modified by Ofek et al. (2011) and Levitan et al. (2011).

The PTF $R$ photometric calibration attempts, within the limits imposed by a survey almost all of whose imaging, especially prior to 2015 , was acquired with only a $R$ filter, to reproduce the SDSS $r^{\prime}$ system. Relative to the reference UCAC-3 astrometric catalog (Zacharias et al. 2010), the astrometric precision of PTF coordinates is about $0{ }^{\prime \prime} 1$ in R.A. and decl.

iPTF, with partial funding from the National Science Foundation (NSF), is in the process of transitioning to the Zwicky Transient Facility (ZTF), to begin operation in late 2017. The $47 \mathrm{deg}^{2}$ field of view of the ZTF camera will be roughly 6 times the area of the PTF camera, and larger than the field of each of the photographic plates used for the Palomar Sky Survey. This, combined with better CCDs with faster readout times, will enable ZTF to observe the sky more than 10 times faster than PTF, while still reaching the same magnitude limit.

\section{Sample Selection}

RR Lyr stand out in a wide-field imaging survey because they are blue and variable. However, the PTF is primarily dedicated to searching for explosive transients. To optimize the cadence for this purpose, almost all PTF imaging until 2014 was carried out with the $R$ filter. Thus, for stellar broadband colors we relied on the SDSS (York et al. 2000). In the SDSS the imaging and thus the derived photometry for a variable star were essentially simultaneous for each of the five filters.

We developed a probabilistic measure of whether or not a star is an RR Lyr variable based largely on its light-curve characteristics. As described briefly in Sesar et al. (2014), we have chosen to use the random forest classifier to isolate a sample of RR Lyr variables from the PTF data. This is a supervised machine-learning algorithm that uses a training

\footnotetext{
6 The PTF Mould $R$ filter is similar in shape to the SDSS $r$-band filter, but shifted $27 \AA$ redward.
} 
sample and a feature set to build a forest of decision trees. Random forest algorithms are able to determine the importance of each feature used for classification, and they are not strongly affected by outliers. Random forest classifiers also tend to be less affected by small changes in the training sample than other classification trees because of the random selection of a subset input features at each node, resulting in a selection that maintains accuracy while reducing correlation (Breiman 2001). They are easily applied to very large sets of time series data (i.e., light curves) and have been used extensively on such data sets in the past few years; see, e.g., Richards et al. (2011), Nun et al. (2014), McCauliff et al. (2015), and Carrasco et al. (2014) for QSOs, and in high-energy physics, see, e.g., Sharma et al. (2014). Because of the algorithm's features and its previous success in classifying variable sources, it was selected as the algorithm of choice for the RR Lyrae classifier.

As a supervised algorithm, the random forest classifier requires a training sample and a set of features. After some experimentation, we settled on 10 features to characterize the light curves, including several suggested by Stetson (1996). The training sample consisted of PTF light curves of RR Lyr stars and non-RR objects in SDSS Stripe 82, where the RR Lyr are identified in Sesar et al. (2010), and the remaining stellar objects are non-RR Lyr stars. Hernitschek et al. (2016) give extensive details on a similar selection applied to the PanSTARRS PV2 (internal process V2) data. The output of the classifier (denoted Pr, range 0-1) is a measure of probability that the light curve under consideration is that of an RR Lyr. The rank ordering of the Pr values is correct, but the conversion to an actual probability has not been quantified. Light-curve parameters are also determined, including the period, amplitude, and epoch of maximum light that defines $\phi=0$.

Experience gained with the random forest classifier suggested that 30 epochs, provided that they are well spaced compared to the typical RR Lyr period, suffice to identify an RR Lyr variable, phase the light curve, and determine its period. We thus require a minimum of 30 detections of a given star for it to be included in the RR Lyr search. Until the fall of 2014, after this sample was originally assembled, the RR Lyrae project had no assigned P48 time. Thus, to assemble our sample of candidate RR Lyr stars, we data-mined the PTF archive in 2013, searching for high galactic latitude fields that had more than $40 R$ images. We ran the random forest classifier on the time series of photometry (ignoring images that yielded only upper limits instead of detections) for all stars in such fields that showed evidence of variability and that had 30 or more detections at $R$. We retained only those with a minimum $\operatorname{Pr}$ of 0.70 and that have reddeningcorrected $g-r$ colors from the SDSS within the range appropriate for RR Lyr, e.g., that of Sesar et al. (2010). As a final check, the NASA/IPAC Extragalactic Database was used to remove known QSOs.

The area on the sky of the Sgr stream within $9^{\circ}$ of the orbital plane of this tidal stream (i.e., $\left|B_{\text {Sgr }}\right|<9^{\circ}$, where $B_{\text {Sgr }}$ is the latitude in the $\mathrm{Sgr}$ stream coordinate system defined in Appendix A of Belokurov et al. 2014) was excluded. Other known streams, compiled recently by Grillmair \& Carlin (2016), are all closer than $50 \mathrm{kpc}$ and hence not relevant here.

Because the RR Lyr survey with PTF and its successors is largely piggybacking on the various SN surveys, our sample probes widely separated randomly selected high galactic latitude pencil beam fields, each $7.3 \mathrm{deg}^{2}$ in size. Substructure

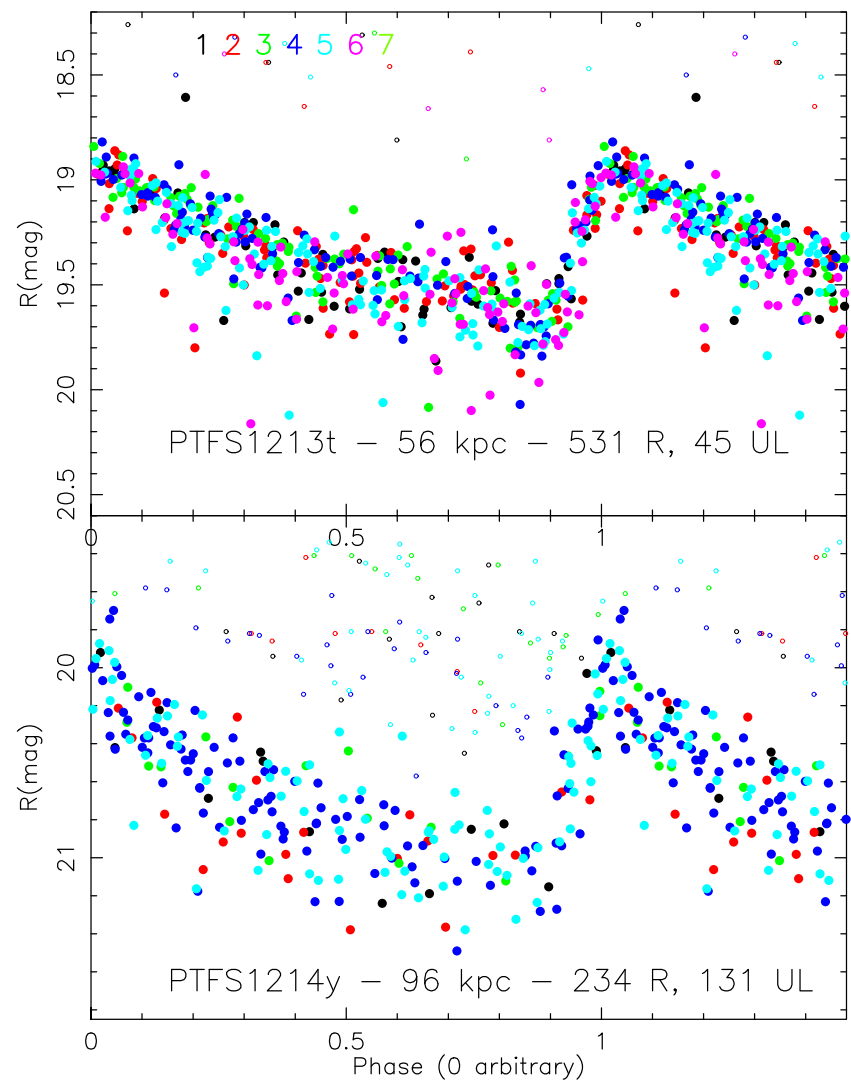

Figure 1. Phased light curve for PTFS1213t (R.A., decl.: 199.472902, $32.118009, r=56 \mathrm{kpc}, 576$ epochs of $R$ imaging, of which 45 are only upper limits, indicated by small open circles) and for PTF1214y (R.A., decl.: $210.61273,39.29614, r=96 \mathrm{kpc}, 365$ epochs of $R$ imaging, of which 131 are only upper limits). The colors denote the years since the first PTF observation; the key for the colors is at the top of the upper panel, black points from the first year, etc.

effects should be minimized because of our sparse sampling over a very large area on the sky.

Figure 1 shows the light curve of one of the brighter RR Lyr in our sample $(r=56 \mathrm{kpc})$, as well as that of one of the more distant RR Lyr $(r=96 \mathrm{kpc})$. The observations extend over more than $6 \mathrm{yr}$, with hundreds of detections in the PTF $R$ filter, and with good phasing throughout. We ignore the Blazhko effect, a modulation with time of the pulsation amplitude seen in some RR Lyr stars, as our light curves in general are not of high enough quality to detect this.

Since the PTF is primarily dedicated to the discovery and study of high-amplitude explosive transients such as $\mathrm{SNe}$, observations are carried out even when sky conditions (seeing or transparency) are not optimal, provided that it is safe to open the dome. This means that the depth and point-source image size for an individual exposure will vary over a wide range as observations of a specific field are accumulated over several years. Many of the PTF images have a limiting magnitude much brighter than that of the median $R=20.6$ mag $(5 \sigma)$ limit. Some PTF images, taken under very good conditions (clear night, excellent seeing, excellent telescope performance) reach deeper than the nominal limit. Thus, the quality of the light curve of a candidate variable star is not just a function of the brightness of the star and the number of epochs available in the PTF archive. This complicates estimating the completeness corrections in our sample. 


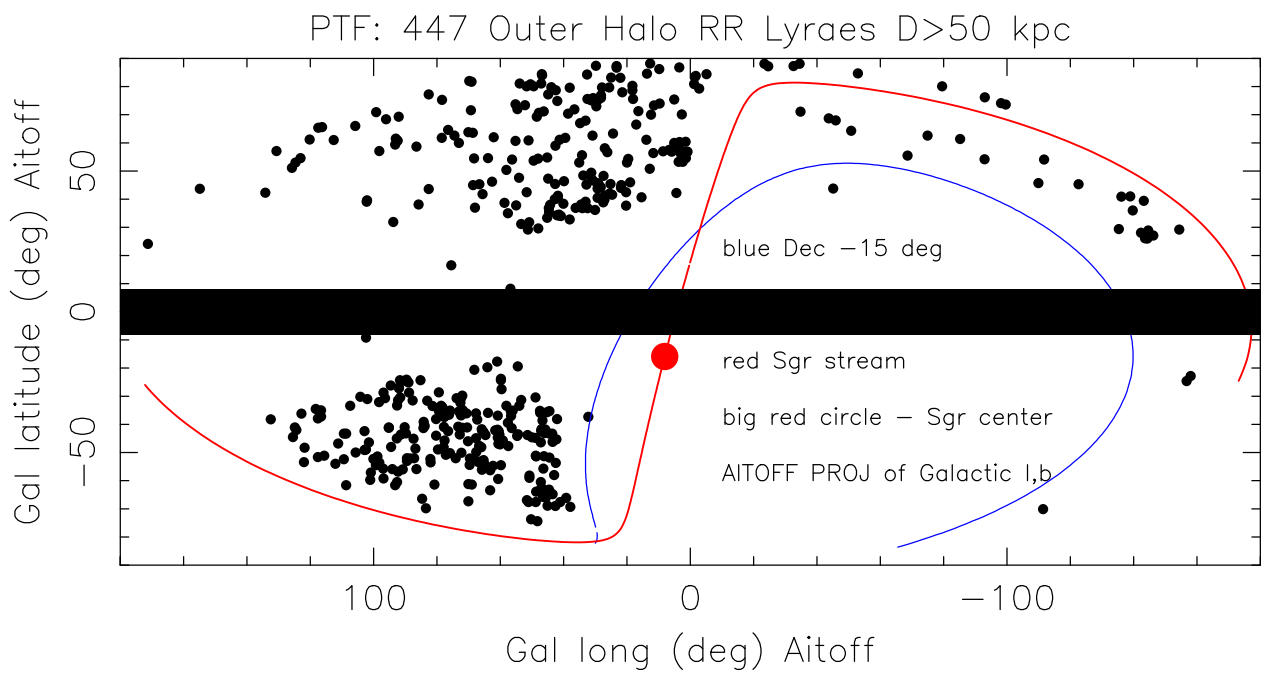

Figure 2. Location on the sky in an Aitoff projection of galactic coordinates of the sample of 447 PTF outer halo candidate RR Lyr stars. The larger points have distances beyond $85 \mathrm{kpc}$. The locus of the Sgr stream is denoted by the red curve. The center of the Sgr galaxy is indicated by the large red dot. Decl. $-15^{\circ}$ is indicated by the blue curve.

We assembled a list of 447 candidate RR Lyr selected from the PTF database to be at a heliocentric distance of $50 \mathrm{kpc}$ or greater at high Galactic latitude, ${ }^{7}$ with SDSS $(g-r)$ colors from DR10 (Ahn et al. 2014) within an appropriate range and outside the Sgr streams. Figure 2 shows their location on the sky using Galactic coordinates. Allowing a distance separation of 5\%, the closest pair of candidate RRab has a separation on the sky of $0.16 \mathrm{kpc}$ and a distance of $50.5 \mathrm{kpc}$. There is one other close pair with separation on the sky of less than $0.4 \mathrm{kpc}$.

Some trends appear within this sample of 447 candidate RR Lyr as shown in Figure 3, including a trend toward higher median amplitude with approximately constant median period at larger distances. There is also a trend of lower Pr (recall that $0.7<\operatorname{Pr}<1.0$ ) toward larger distances; the median probability index decreases from 0.92 for the first bin $(r=50-53 \mathrm{kpc})$ shown in Figure 3 to 0.82 for the last bin $(r>94 \mathrm{kpc})$. These trends are not surprising given that we are approaching the limiting magnitude of the PTF survey at the largest distances probed.

As will be described later, 112 stars were selected for spectroscopic observations. These consist of the higherprobability RR Lyr candidates from this list, within the constraints imposed by the specific dates of the assigned telescope time.

\section{Distances}

RRab are almost standard candles, and we adopt a median for their extinction-corrected $M_{R}$ mag (averaging the flux of the best-fitting light-curve template over one period) of $+0.6 \mathrm{mag}$. However, it is well known that there is a small dependence of luminosity on period (linear in $\log P$ ) and on metallicity (linear in $[\mathrm{Fe} / \mathrm{H}])$. We first assess the range in period of RR Lyr stars. We use the sample of $173 \mathrm{RRab}$ isolated by Sesar et al. (2010) in Stripe 82 of the SDSS with mean $R$ fainter than 17.0 mag. This sample has excellent photometric data with many observed epochs. It covers a wide range in distance and should be representative of our sample as well. Figure 4 presents the

\footnotetext{
7 The minimum heliocentric distance of $r=50 \mathrm{kpc}$ for a star in our sample corresponds to a galactocentric distance between 45.9 and $57.8 \mathrm{kpc}$ depending on $(l, b)$.
}

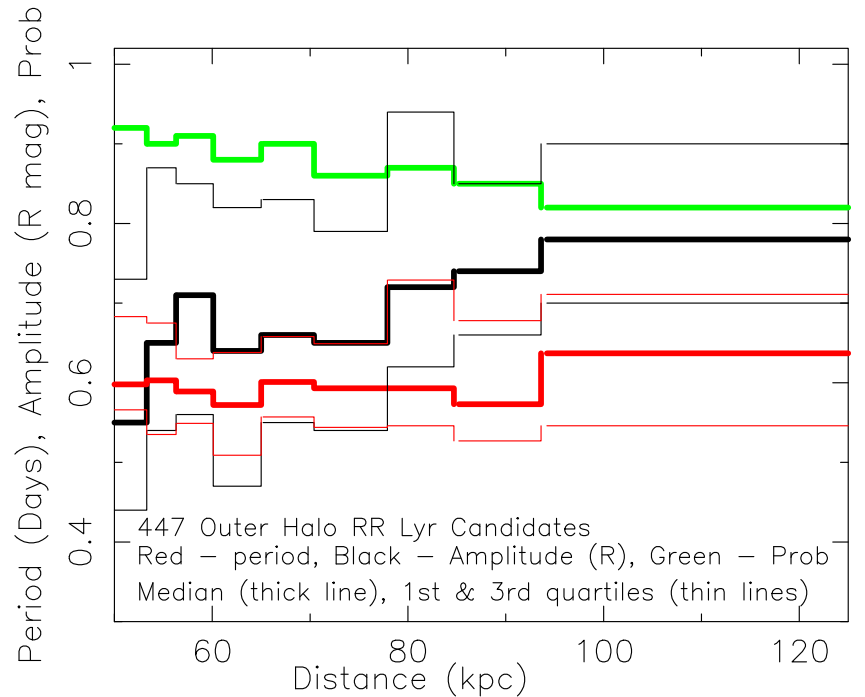

Figure 3. Median, first, and third quartiles of periods and of amplitudes of our sample of 447 RR Lyr candidates beyond $50 \mathrm{kpc}$ are shown in nine distance bins. The median probability is also shown for each bin. The first bin contains 47 RR Lyr, while the more distant ones each contain 50 variables.

period-amplitude relation for these stars. The RR type $c$ variables from the Stripe 82 sample are also shown in this figure. Note that they have have shorter periods and lower amplitudes than do the RRab.

A histogram of the periods for the $173 \mathrm{RRab}$ stars in Stripe 82 with $R$ fainter than $17.0 \mathrm{mag}$ from the sample of Sesar et al. (2010) is shown in the lower panel of Figure 5; the upper panel displays the same for our PTF RRab sample. The values for mean and rms dispersion of each of the two samples are indicated in the figure; they are essentially identical, which is gratifying, as both probe deep into the outer halo of the MW.

We correct for the period term in the luminosity of RRab adopting the coefficient given by Marconi et al. (2015), who present theoretical period-luminosity relations for RR Lyr stars over a range of metallicity based on their new nonlinear timedependent convective hydrodynamical models of RR Lyr stars. These supersede earlier calculations by Chaboyer (1999), Caceres \& Catelan (2008), and others. We note that their 


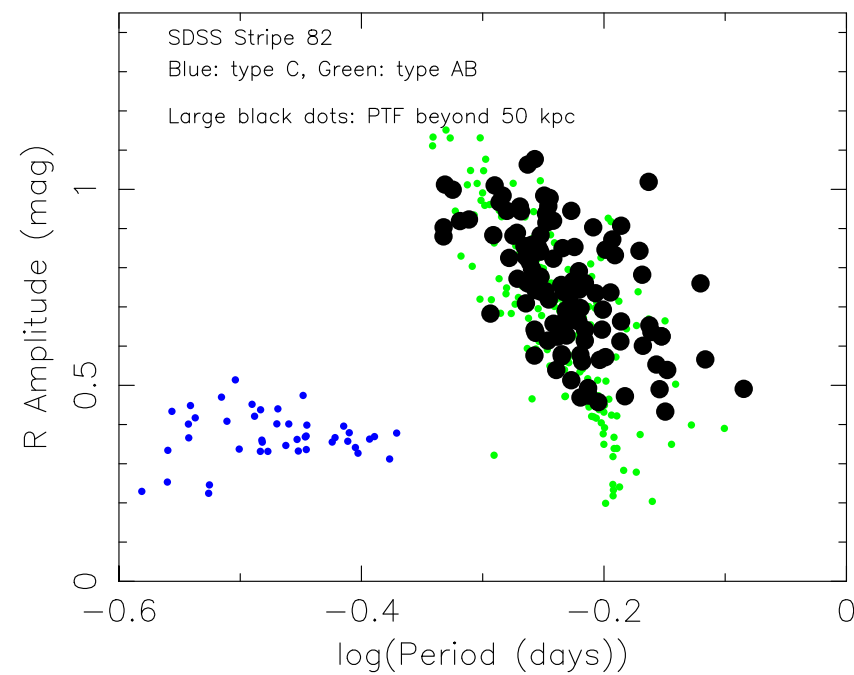

Figure 4. Period-amplitude relation for the 112 Keck PTF outer halo RR Lyr stars (large black points), as well as that of the SDSS Stripe 82 sample from Sesar et al. (2010) (type $a b$ in green, type $c$ in blue).

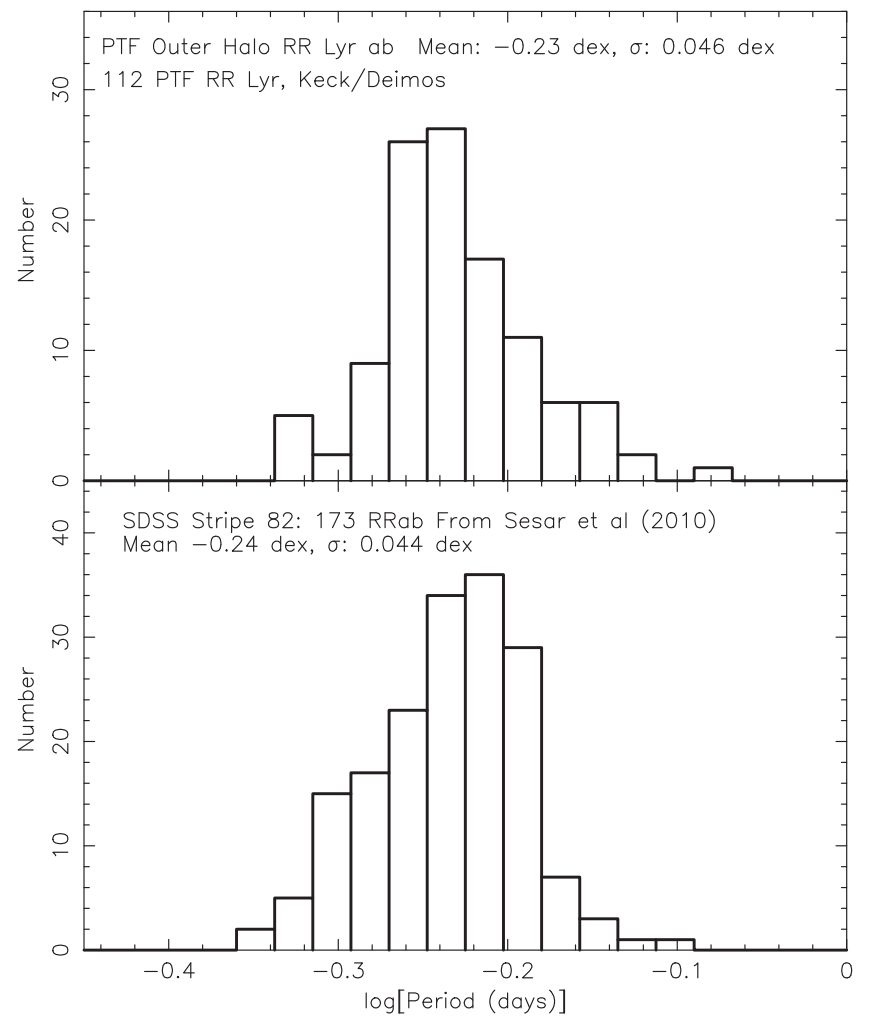

Figure 5. Histogram of $\log$ (period) for our Keck $v_{r}$ sample (upper panel) and that of the 173 RR Lyr $a b$ sample from Sesar et al. (2010) (lower panel).

dependence on $\log (P)$ for fundamental-mode RR Lyr in the $i$ band is 1.6 times larger than that of Caceres \& Catelan (2008), and therefore we may hope that use of their coefficients will provide an upper limit to the change in mean $R$ with both $P$ and $[\mathrm{Fe} / \mathrm{H}]$.

The luminosity dependence on the period, which is $-1.39 \log (P)$, is in general small, as the median period for our outer halo RR Lyr sample is 0.553 days with $\sigma \log (P)=0.044$ dex. This results in a change in distance of less than $3 \%$; even at the extreme high and low values of log $(P), P=0.456$ days and $P=0.793$ days, the resulting change in distance incurred by including the period-dependent term does not exceed $10 \%$. Note that the sample of RRab in SDSS Stripe 82 studied by Sesar et al. (2010) has a median period of 0.582 days and a $\sigma$ for $\log (P)$ of 0.044 dex, almost identical to that of our outer halo sample.

Corrections for interstellar absorption were applied based on the reddening map of Schlegel et al. (1998). If a reddening map with larger extinction at high galactic latitude is used, the distances to the RR Lyr would increase.

The absolute luminosity of RRab also depends on the metallicity, for which $[\mathrm{Fe} / \mathrm{H}]$ is used. Schorck et al. (2009) have established the metallicity distribution of the outer halo for very low metallicities; the fraction of the stellar content of the MW halo that is extremely metal-poor is very small. Again using the coefficients of Marconi et al. (2015), we find that potential variations of $[\mathrm{Fe} / \mathrm{H}]$ of $\pm 0.5 \mathrm{dex}$ about a (low, but not extremely low) mean metallicity lead to an uncertainty in the distance of $4 \%$.

Another key issue is the accuracy of the mean $R$ mag measured from our light curves. Although the uncertainty of an $R$ measurement at a single epoch may be large, up to $0.2 \mathrm{mag}$, the mean $R$ will be much more accurate. A reasonable estimate of this, particularly for stars with many epochs (ignoring upper limits) in their light curves, is $0.03 \mathrm{mag}$, which corresponds to a distance uncertainty of $1.5 \%$.

Thus, if one assumes that the mean metallicity in the outer halo beyond $50 \mathrm{kpc}$ is low and only has a modest gradient with distance and a modest range at any outer halo location, which seems appropriate for the outer halo excluding the Sgr streams, then based on the uncertainties found above, our distances for RRab with good light curves should be precise to $5 \%$. Here the dominant term results from the unknown metallicity. Lightcurve quality for these RRab will improve with additional observations once ZTF is commissioned, resulting in better light curves with more detections.

An empirical test of how large (actually how small) the distance errors might be for RR Lyr owing to their range of periods was carried out by B. Sesar. Using the Pan-STARRS RR Lyr catalog (to become publicly available on 2017 November 1), he calculated the dispersion in distance based on assuming a fixed absolute $R$ mag, ignoring the period and metallicity dependences, for a large $(\sim 200)$ sample of RRab in the Draco dSph galaxy. He measured an rms scatter of $0.08 \mathrm{mag}$, corresponding to a distance precision of $4 \%$ for this sample. In Section 3.3 of Sesar et al. (2017c) this test of calculating the dispersion in distance is extended to two additional dSph satellites of the MW, Sextans, and Ursa Minor, again with excellent results. In these tests the metallicity dependence within each of these $\mathrm{dSph}$ galaxies was ignored. The metallicity range within Draco extends from $[\mathrm{Fe} / \mathrm{H}]=$ -3.0 to -1.5 dex (Cohen \& Huang 2009; Kirby et al. 2011), and the range within Ursa Minor is similar (Cohen \& Huang 2010; Kirby et al. 2011). Thus, the metallicity dependence is not a significant contributor for most, if not all, outer halo stars. It is clear that distances accurate to $5 \%$ can be obtained for RRab with high-quality light curves.

The above discussion does not address the issue of the the uncertainty in the adopted extinction map. The recent extinction coefficients of Schlafly \& Finkbeiner (2011) (their Table 6) and the Schlafly et al. (2014) dust map lead to somewhat higher extinction at high Galactic latitude than that we adopt, which would result in our distances being slightly 
Table 1

Light-curve Parameters for RR Lyr Candidates

\begin{tabular}{|c|c|c|c|c|c|c|c|c|}
\hline $\begin{array}{l}\text { R.A. } \\
\text { (deg) }\end{array}$ & $\begin{array}{l}\text { Decl. } \\
\text { (deg) }\end{array}$ & $\begin{array}{l}\text { Period } \\
\text { (days) }\end{array}$ & $\begin{array}{c}0 \text { Phase }^{\mathrm{a}} \\
\text { (days) }\end{array}$ & $\begin{array}{c}\text { Amp } \\
(R \mathrm{mag})\end{array}$ & $\begin{array}{c}\text { Mean } R \\
\text { (mag) }\end{array}$ & $N(R)^{\mathrm{b}}$ & $N(g)^{\mathrm{c}}$ & Quality $^{\mathrm{d}}$ \\
\hline 3.77632 & 28.37604 & 0.7038231 & 56917.70 & 0.62 & 20.64 & 39 & 3 & 2 \\
\hline 10.51398 & 15.64457 & 0.6035999 & 55473.75 & 0.70 & 19.94 & 44 & 131 & 1 \\
\hline 13.31085 & 17.13101 & 0.6009332 & 55477.85 & 0.66 & 19.64 & 59 & 0 & 1 \\
\hline 21.20049 & 20.43072 & 0.5733125 & 56239.63 & 0.66 & 20.31 & 82 & 16 & 1 \\
\hline 21.39914 & 3.82265 & 0.6442016 & 55477.74 & 0.83 & 19.37 & 483 & 78 & 1 \\
\hline 22.09602 & 13.81008 & 0.5529058 & 55906.68 & 0.58 & 19.12 & 158 & 0 & 1 \\
\hline 358.89981 & 34.25303 & 0.5126550 & 56256.68 & 1.01 & 19.27 & 73 & 197 & 1 \\
\hline Probable & Sgr & Stream & & & & & & \\
\hline 185.831403 & 11.011716 & 0.5416933 & 55899.95 & 0.76 & $\ldots$ & $\ldots$ & $\ldots$ & $\ldots$ \\
\hline 189.730895 & 7.902692 & 0.4639002 & 55378.71 & 1.17 & $\ldots$ & 287 & 69 & 1 \\
\hline 191.481527 & 5.967331 & 0.6101711 & 55333.68 & 0.55 & $\ldots$ & 280 & 116 & 1 \\
\hline 208.767863 & 5.213217 & 0.4890867 & 55330.74 & 1.08 & $\ldots$ & 304 & 114 & 1 \\
\hline
\end{tabular}

Notes.

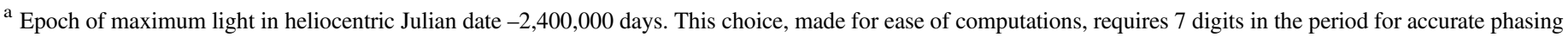
at the present epoch.

${ }^{\mathrm{b}}$ Number of epochs taken with the PTF $R$ filter in which the star was detected as of late 2016.

c Number of epochs taken with the PTF $g$ filter in which the star was detected as of late 2016.

d Observed $R$ light curve resembles that of an RR Lyr: 1-excellent; 2-probable; 3-uncertain.

(This table is available in its entirety in machine-readable form.)

underestimated. Furthermore, we have adopted an absolute $R$ mag (corrected for reddening) for the median of our sample of +0.6 mag. If this choice is incorrect, all of our distances need to be scaled appropriately.

\section{Light-curve Quality}

Table 1 gives the light-curve parameters for each star in our sample of 112 RRab in the Keck $v_{r}$ sample. The last three columns of this table indicate for each RRab the number of available $R$-band detections, the number of $g$-band detections, and an assessment of the light-curve quality in the PTF Stellar Light Curve database as of late 2016. The latter was set by visual inspection by the first author in late 2016. Quality 1 light curves are clearly RR Lyr variables, quality 2 are probably RR Lyr, while the nature of objects with quality 3 light curves is uncertain.

The initial determination of light-curve parameters for the Keck $v_{r}$ sample was carried out in 2014, at which time the number of available epochs was smaller (often considerably smaller) than at present. Beginning in late 2014 we were allocated a total of roughly $100 \mathrm{hr}$ of P48 (i.e., PTF) time made available through the Caltech allocation to improve the light curves of the more distant stars in the Keck $v_{r}$ sample. These distant stars have a much higher fraction of nondetections than do the brighter end of our sample, and so we need additional imaging to raise the number of detections to a level that ensures accurate characterization of the light-curve parameters. Our goal in this effort is 100 detected epochs of $R$-band imaging for each of the most distant RR Lyr candidates.

In late 2016 and early 2017 the light curve of each of the stars in the Keck $v_{r}$ sample was checked to look for problems in the phasing, i.e., incorrect periods or determinations of $\phi=0$ caused by the limited data available when the light curves were first determined in 2014. As necessary, the light-curve parameters were redetermined at that time, and the correction from the observed $v_{r}$ to the systemic $v_{r}$ described in Section 6 was updated using the new ephemeris parameters. This was a crucial step, as the initial values were in several cases sufficiently far off that the accumulated phase change over several years significantly affected the derived phase correction to the observed $v_{r}$.

At the present time, as indicated in Table 1, only 12 stars from the Keck $v_{r}$ sample of 112 stars have less than 50 detections in $R$ or 50 detections in the $g$ filter. Only 4 stars (4\%) out of the total sample of 112 RR Lyr are classified as having poor light curves (i.e., quality 3 ). As described earlier, we have until very recently had no control over the observing plan for $\mathrm{PTF} / \mathrm{P} 48$ time, which is defined by the other major projects of the PTF, especially the SN projects. Thus, the number of epochs of observation of a given star varies from a minimum of $\sim 30$ up to $\sim 700$ when an RR Lyr candidate is by chance located in a field that is of major interest to one of the other PTF projects.

\section{Radial Velocity Measurements}

A spectroscopic campaign to obtain radial velocities for RR Lyr candidates began at the Keck Observatory with the Deimos spectrograph (Faber et al. 2003) in the spring of 2014 following a brief effort to use the Double Spectrograph (DBSP) on the Hale Telescope at the Palomar Observatory, which yielded one useful spectrum. RRab are pulsating periodic variable stars. Spectroscopic observations to determine $v_{r}$ must be taken within the range of phase such that $\left(d v_{r} / d t\right)$ in the stellar atmosphere is as small as possible. The observing list for each night was compiled from the candidate RRab stars near the meridian during the night with the appropriate range of phase $(\phi=0.1-0.7)$ accessible during that time. Observation planning therefore required having both a coordinate list and accurate predicted phases from the start to the end of the night for the specific date; the phases are calculated from the periods we determined from the PTF light curves. Candidates with high probability index $(\operatorname{Pr}>0.9)$ were favored, but it was sometimes necessary to incorporate candidates with lower Pr to fill in gaps in the observing plan for a specific night. 


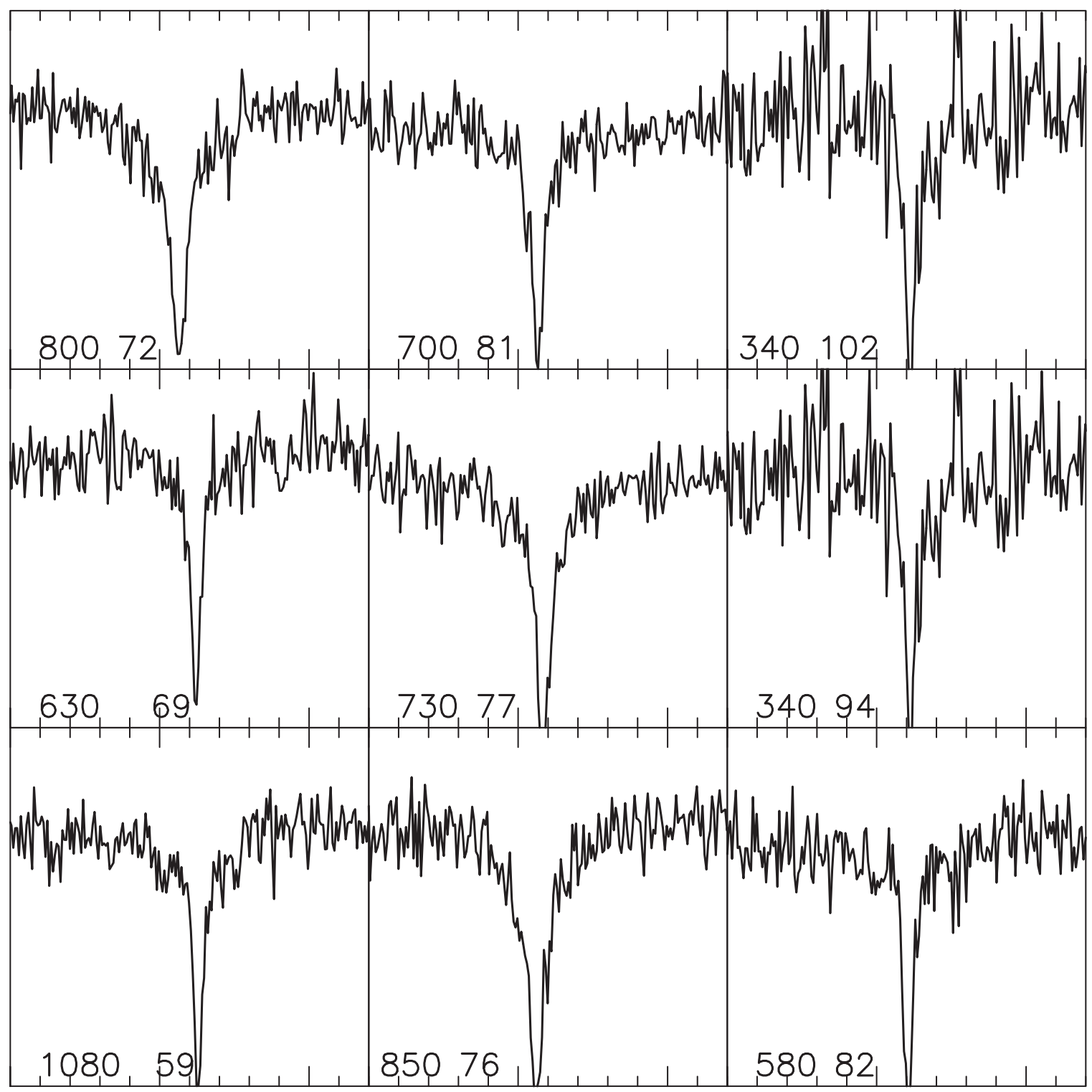

Figure 6. Plots of the 1D extracted spectra for nine RR Lyr from our sample. The vertical scale of each panel ranges from 1.2 times the continuum signal near H $\alpha$ to 0.5 times the continuum signal. The text at the bottom of each panel gives the continuum signal level and the distance. The panels are ordered by the distance from 59 (bottom left) to $102 \mathrm{kpc}$ (upper right). The wavelength range ( $X$-axis) of each panel is $6500-6620 \AA$.

The Deimos spectrograph was usually configured with the 600 groove $\mathrm{mm}^{-1}$ grating blazed at $7500 \AA$ and spectral resolution $\sim 2000$ for a 1.0 arcsec wide slit and a scale of $0.65 \AA$ pixel $^{-1}$. Spectra were taken with the central wavelength set to $7500 \AA$. Most spectra were taken with a 1.0 arcsec wide slit, but on nights with good seeing, the 0.7 or the 0.8 arcsec slit was used, yielding correspondingly higher spectral resolution. During our first Keck run, a small number of spectra were acquired with the 1200 groove $\mathrm{mm}^{-1}$ gold-coated grating blazed at $7545 \AA$, which yielded even higher spectral resolution. However, the velocity precision for an exposure of a fixed time turned out not to be better than with the 600 groove $\mathrm{mm}^{-1}$ grating owing to the increased signal-to-noise ratio $(\mathrm{S} / \mathrm{N})$ with the 600 groove $\mathrm{mm}^{-1}$ grating. The maximum (and typical) exposure time was set to 30 minutes to avoid excessive phase blurring. Figure 6 shows spectra in the region of $\mathrm{H} \alpha$ for nine of our RR Lyr candidates selected to cover the full range in distance of our sample. Note the degradation in the $\mathrm{S} / \mathrm{N}$ at the largest distances, which arises from the fixed maximum integration time of 30 minutes.

The determination of the systemic $v_{r}$ for an RR Lyr requires knowledge of the phase at the time of the observation. A correction that depends on the phase is applied to get the systemic $v_{r}$, then a heliocentric correction is applied, and finally we apply a correction to the galactocentric rest frame (Galactic standard of rest (GSR)).

The uncertainties in the systemic velocities include both a measurement error and a term for the uncertainty in fitting to the model radial velocity curve. Details for this calculation are given in Section 5.3 of Sesar et al. (2012). Since typical $v_{r}$ amplitudes over the period for RR Lyr stars of $\mathrm{H} \alpha$ are $\sim 110 \mathrm{~km} \mathrm{~s}^{-1}$, it is important that the phase of observation be determined accurately. This requires accurate periods and phasing.

The primary feature we use for $v_{r}$ determination is $\mathrm{H} \alpha$. Due to the low efficiency of Deimos in the blue, we do not achieve an $\mathrm{S} / \mathrm{N}$ high enough there to use the higher Balmer lines or the 
Table 2

$v_{r}$ for RR Lyr Candidates

\begin{tabular}{|c|c|c|c|c|c|c|}
\hline $\begin{array}{l}\text { R.A. } \\
\text { (deg) }\end{array}$ & $\begin{array}{l}\text { Decl. } \\
\text { (deg) }\end{array}$ & $\begin{array}{c}\text { Distance }^{\mathrm{a}} \\
(\mathrm{kpc})\end{array}$ & No. Spectra & $\begin{array}{c}v_{r}^{\mathrm{b}} \\
\left(\mathrm{GSR} \mathrm{km} \mathrm{s}^{-1}\right)\end{array}$ & $\begin{array}{c}\sigma\left(v_{r}\right) \\
\left(\mathrm{km} \mathrm{s}^{-1}\right)\end{array}$ & Date \\
\hline 3.77632 & 28.37604 & 109.1 & 1 & 212.7 & 21.0 & 2015 Sep \\
\hline 10.51398 & 15.64457 & 75.6 & 1 & -240.2 & 17.7 & 2016 Sep 5 \\
\hline 13.31085 & 17.13101 & 65.9 & 1 & -131.1 & 18.4 & 2015 Oct \\
\hline 21.20049 & 20.43073 & 88.3 & 1 & -124.4 & 18.4 & 2015 Oct \\
\hline 21.39914 & 3.82266 & 59.2 & 1 & 20.4 & 19.7 & 2015 Oct \\
\hline 22.09602 & 13.81008 & 50.5 & 1 & -21.2 & 17.8 & 2015 Oct \\
\hline 358.89981 & 34.25304 & 53.1 & 1 & -55.9 & 20.9 & 2015 Oct \\
\hline Sgr & Stream ? & & & & & \\
\hline 185.831403 & 11.011716 & 86.9 & 1 & -62.5 & 20.5 & 2014 Apr \\
\hline 189.730895 & 7.90269 & 86.1 & 1 & -120.2 & 25.5 & 2014 Apr \\
\hline 191.481527 & 5.967331 & 79.5 & 1 & 4.1 & 19.2 & 2014 May \\
\hline 208.767863 & 5.213217 & 83.9 & 1 & -16.4 & 29.0 & 2014 Apr \\
\hline
\end{tabular}

Notes.

a Heliocentric distance.

b $v_{r}$ corrected to the systemic velocity, then to the heliocentric velocity, and then to the Galactic system of rest.

c Two Keck/Deimos spectra have been taken. See Table 3 for details.

d A DBSP spectrum taken with the Hale Telescope at Palomar Observatory.

(This table is available in its entirety in machine-readable form.)

strong blue metallic lines. Sesar (2012) has derived template velocity curves that calibrate the change in velocity as a function of pulsation phase for several of the Balmer lines; we adopt his $\mathrm{H} \alpha$ template here (see also the very detailed recent study by Chadid et al. 2017). The normalized $v_{r}-\phi$ curves of Sesar (2012) are then scaled by the amplitude of variation of the light curve to derive the correction from the observed $v_{r}$ at phase $\phi$ to the systemic velocity. The other major features clearly visible in these spectra are the Paschen lines around $8600 \AA$, but we are not aware of any $v_{r}-\phi$ calibration for them. The infrared $\mathrm{Ca}$ triplet and a few O I lines are also visible in these spectra, and we will undertake an attempt to use them as metallicity indicators in the future.

Standard arc lamps ( $\mathrm{Ne}, \mathrm{Ar}, \mathrm{Kr}$, and $\mathrm{Xe}$ ) were used for wavelength calibration, which was then tuned up slightly for each observation of an RR Lyr candidate using the night-sky emission lines superposed on each stellar spectrum, important as there are few arc lines in the region of $\mathrm{H} \alpha$, the primary feature we are using to determine $v_{r}$.

From our Keck runs beginning in 2014 April and extending through 2016 September we have acquired spectra of roughly 135 candidate RR Lyr from our PTF sample, 112 of which we believe to be RRab stars based on their light curves, their colors, and their spectra, the remainder being a few quasars, a few RR type $c$ variables, or other types of variable stars. The stars in the Keck $v_{r}$ sample are widely dispersed on the sky, with the closest pair in the list of candidate RRab stars being separated by 0.2 . Thus, no multiplexing was possible, and a substantial number of Keck nights were required to obtain this set of spectra.

The set of 112 RR Lyr with spectroscopic $v_{r}$, which are listed in Table 1, have heliocentric distances of $50<r<$ $109 \mathrm{kpc}$ with a median of $r=73 \mathrm{kpc}$. The resulting $v_{r}$ relative to the GSR and its uncertainty are given in Table 2. Typical uncertainties for $v_{r}$ from a single measurement range from 17 to $20 \mathrm{~km} \mathrm{~s}^{-1}$.

A separate list of four RR Lyr that were observed during our first run with Deimos on Keck for this project, but which are probably part of the Sgr stream, is given at the end of this table.
The exclusion region for the Sgr stream was originally set to be within $5^{\circ}$ of the orbital plane of this tidal stream, but it was raised to $9^{\circ}$ shortly after observing commenced. Once the exclusion region around the Sgr stream was increased in size, these four stars were dropped from our sample of candidate RR Lyr in the outer halo of the Galaxy. There are seven stars in our sample that are between $9^{\circ}$ and $15^{\circ}$ from the orbital plane of the Sgr tidal stream.

\subsection{Test of $v_{r}$ Accuracy}

To demonstrate the accuracy of our systemic $v_{r}$ for RR Lyr variables, Table 3 gives the independent $v_{r}$ for those candidate RR Lyr variables from our sample with more than one Deimos spectrum; there are $22\left(\sim 20 \%\right.$ of the total sample with Keck $\left.v_{r}\right)$ with two independent spectra. In several cases, the two Deimos spectra were taken on the same night, often consecutively, but analyzed independently. The agreement between the two determinations of $v_{r}(\mathrm{GSR})$ for each of these six stars is good. There are 11 stars with two spectra from the same night or from consecutive nights. These in general show small differences in $v_{r}$ between the two spectra. Only three have differences exceeding $20 \mathrm{~km} \mathrm{~s}^{-1}$, with the largest difference being $33 \mathrm{~km} \mathrm{~s}^{-1}$. Given that the nominal uncertainty of a single measurement is $\sim 20 \mathrm{~km} \mathrm{~s}^{-1}$, this agreement is good.

There are eight RR Lyr candidates with two Deimos spectra taken more than a year apart. The differences are larger here, ranging from 9 to $51 \mathrm{~km} \mathrm{~s}^{-1}$, with two having differences exceeding $40 \mathrm{~km} \mathrm{~s}^{-1}$.

The difference in $v_{r}$ for stars with multiple spectra is shown in Figure 7 as a function of the separation in time between the two epochs of observation, which increases along the $X$-axis. As indicated above, for small differences in time, the difference between the two $v_{r}$ for a given star is close to or within the expected uncertainties, but once the time interval becomes large (months to years), there are two cases with disagreements exceeding $40 \mathrm{~km} \mathrm{~s}^{-1}$ between the two derived $v_{r}$.

We suspect that these disagreements arise in part from possible errors in the phases due to uncertainties in the period. Thus, our process to determine $v_{r}$ in the GSR for these RR Lyr 
Table 3

$v_{r}$ for RR Lyr Candidates with Two Keck Deimos Spectra

\begin{tabular}{|c|c|c|c|c|}
\hline R.A. & $\begin{array}{l}\text { Decl. } \\
(2000)\end{array}$ & $\begin{array}{c}v_{r}^{\mathrm{a}} \\
\left(\mathrm{GSR}: \mathrm{km} \mathrm{s}^{-1}\right)\end{array}$ & $\begin{array}{c}\sigma\left(v_{r}\right)^{\mathrm{b}} \\
\left(\mathrm{km} \mathrm{s}^{-1}\right)\end{array}$ & Date \\
\hline \multicolumn{5}{|l|}{ Same Night } \\
\hline \multirow[t]{2}{*}{135.734329} & 61.632797 & -1.6 & 18.5 & 2016 Apr 5 \\
\hline & & 24.7 & 18.5 & 2016 Apr 5 \\
\hline \multirow{2}{*}{204.352357} & 38.228202 & -111.6 & 19.1 & 2016 Apr 5 \\
\hline & & -110.3 & 19.1 & 2016 Apr 5 \\
\hline \multirow[t]{2}{*}{205.905884} & 32.5560532 & 109.1 & 17.2 & 2014 Apr 30 \\
\hline & & 107.7 & 17.2 & 2014 Apr 30 \\
\hline \multirow[t]{2}{*}{323.072937} & -3.48319101 & -6.0 & 20.6 & 2015 Oct 14 \\
\hline & & 26.5 & 20.6 & 2015 Oct 14 \\
\hline \multirow[t]{2}{*}{329.612610} & 15.671644 & -217.9 & 20.3 & 2015 Oct 13 \\
\hline & & -203.9 & 20.3 & 2015 Oct 13 \\
\hline \multirow[t]{2}{*}{332.410919} & 18.2443104 & -67.6 & 18.1 & 2015 Oct 13 \\
\hline & & -77.5 & 18.1 & 2015 Oct 13 \\
\hline Consecutive & Nights & & & \\
\hline \multirow[t]{2}{*}{198.013824} & 37.5026283 & 109.1 & 17.2 & 2014 Apr 30 \\
\hline & & 88.7 & 17.6 & 2014 May 1 \\
\hline \multirow[t]{2}{*}{205.205704} & 36.8539162 & 47.3 & 19.0 & 2014 Apr 30 \\
\hline & & 51.0 & 19.1 & 2014 May 1 \\
\hline \multirow[t]{2}{*}{207.652344} & 44.8125725 & -89.9 & 22.1 & 2014 Apr 30 \\
\hline & & -57.1 & 21.0 & 2014 May 1 \\
\hline \multirow[t]{2}{*}{239.070038} & 36.4328651 & -181.5 & 22.7 & 2014 Apr 30 \\
\hline & & -169.8 & 19.7 & 2014 May 1 \\
\hline \multirow[t]{2}{*}{246.976883} & 31.5548630 & 84.4 & 23.0 & 2014 Apr 30 \\
\hline & & 79.2 & 23.2 & 2014 May 1 \\
\hline \multicolumn{5}{|l|}{$\sim 1$ Month Apart } \\
\hline \multirow[t]{2}{*}{26.332462} & 29.490912 & -43.3 & 18.2 & 2015 Sep 19 \\
\hline & & -32.8 & 19.0 & 2015 Oct 11 \\
\hline \multirow[t]{2}{*}{242.537582} & 21.5110741 & -183.8 & 18.9 & 2016 Jun \\
\hline & & -196.6 & 18.9 & 2016 Jul \\
\hline \multirow[t]{2}{*}{258.777034} & 37.911508 & -94.1 & 20.0 & 2016 Jun \\
\hline & & -72.1 & 20.0 & 2016 Sep 5 \\
\hline Nights Separated by & a Year or More & & & \\
\hline \multirow[t]{2}{*}{191.654206} & 31.937550 & -22.8 & 19.8 & 2016 Apr 30 \\
\hline & & -74.3 & 23.0 & 2014 May 29 \\
\hline \multirow[t]{2}{*}{210.637741} & 38.2323532 & -55.3 & 20.9 & 2014 Apr 30 \\
\hline & & -66.6 & 19.1 & 2016 Apr 5 \\
\hline \multirow[t]{2}{*}{223.622803} & 35.9652824 & 25.4 & 18.3 & 2014 May 18 \\
\hline & & 5.6 & 18.3 & 2016 Jun 4 \\
\hline \multirow[t]{2}{*}{236.473862} & 58.0700874 & 20.7 & 27.7 & 2014 Apr 30 \\
\hline & & 46.2 & 20.2 & 2016 Jun 4 \\
\hline \multirow[t]{2}{*}{239.771881} & 38.5657883 & 48.9 & 17.0 & 2014 May 28 \\
\hline & & 80.5 & 17.0 & 2016 Jun 4 \\
\hline \multirow[t]{2}{*}{253.129211} & 25.3644562 & 78.2 & 19.0 & 2014 Apr 30 \\
\hline & & 87.7 & 19.0 & 2016 Jun 4 \\
\hline 257.613861 & 20.8844376 & -65.0 & 18.8 & 2015 Sep \\
\hline & & -27.0 & 18.8 & 2016 Apr \\
\hline 321.292572 & 4.89428520 & -140.7 & 20.4 & 2015 Oct \\
\hline & & -96.9 & 20.4 & 2016 Jul \\
\hline
\end{tabular}

Notes.

a $v_{r}$ corrected to the systemic velocity, then to the heliocentric velocity, and then to the Galactic system of rest.

b The 1 ouncertainty in the GSR radial velocity.

variables from the observed $v_{r}$ using phase-dependent corrections appears to be working reasonably well in general. However, for a small fraction of our candidate RR Lyr variables, this is not the case. While there are a few unexpectedly large discrepancies, they are relatively small compared to the velocity dispersion among our $112 \mathrm{RR}$ Lyr sample to be discussed later in Section 7.

As indicated earlier, if the star were not a genuine RRab variable or the key light-curve parameters (period and epoch of zero phase) we derived were wrong, incorrect $v_{r}$ would be 


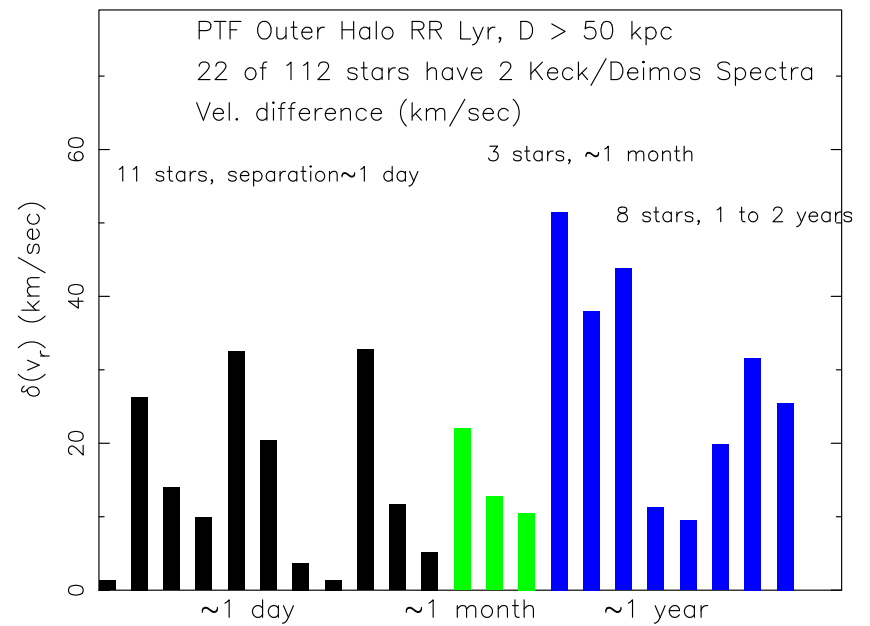

Figure 7. Sample of 22 stars with two Keck/Deimos spectra. The vertical axis is the absolute value of the difference in $v_{r}(\mathrm{GSR})$ for each star with more than one spectrum. The horizontal axis sorts the pairs in order of increasing separation in time between the two observations, with difference ranging from $\sim 1$ day to $\sim 1 \mathrm{yr}$.

derived. However, we note that one of the stars with two independent spectra that shows an unexpectedly large $\delta\left(v_{r}\right)$ has an excellent light curve with 224 detections with the PTF $R$ filter.

\subsection{Contaminants in the Sample of Candidate RR Lyr Stars}

The only blue point sources seen at high galactic latitude are RR Lyr, QSOs, blue horizontal branch (BHB) stars, and blue stragglers. Since our primary selection is by variability resembling that expected for an RRab, the nonvariable BHB stars and blue stragglers become irrelevant. Thus, the primary source of contamination is expected to be QSOs, but the timescale and characteristics of their variation are quite different from those of RRab. As the number of observed epochs of photometric monitoring increases (and this will grow with time as the iPTF transitions into the ZTF), the fraction of contaminating quasars will fall, because their extended light curves will diverge more and more from those of RRab, the variation will not be periodic, etc.

Table 4 lists the five broad-lined objects (i.e., QSOs) we have found from our spectroscopic campaign that are not included in NED. Given that most QSOs are eliminated, as they do not have light curves that resemble RR Lyr, and adding in a check with NED, the contamination rate of QSOs within the sample selected for spectroscopy can be kept very low and can, as described above, be expected to fall with time as the survey time coverage increases. WISE colors (Wright et al. 2010) can also be used to cut down the fraction of QSO contamination (Nikutta et al. 2014) due to the difference in spectral slope between a power law and a (hot) thermal spectrum, but they are of limited use for such distant objects, as they are often so faint that only the W1 color is given in the WISE catalog.

The other potential contaminant of our sample of RRab stars is overtone pulsators, i.e., type $c$ RR Lyr. Several of the variable stars listed at the end of Table 4 are probably RR Lyr $c$. They were originally believed to be RRab and were part of the spectroscopic sample, but as their light curves built up with time, they became inconsistent with the period range and/ or shape appropriate for RRab and were removed. Given the mean difference in luminosity at $R$ of $\sim 0.25$ mag between RR Lyr pulsators in the fundamental and the first-overtone modes,
Table 4

RR Lyr Candidates That Are Not RR Lyr(ab)

\begin{tabular}{|c|c|c|c|}
\hline R.A. & Decl. & PTF $R$ & Comments \\
\hline QSO & (not in NED & $\begin{array}{c}\text { as of } \\
2016 \mathrm{Jul})\end{array}$ & \\
\hline 3.776320 & 28.376036 & 20.6 & Broad em. $5650 \AA$ \\
\hline 22.518406 & 5.325709 & 20.1 & Broad em. 6200, $9200 \AA$ \\
\hline 67.040609 & 0.551808 & 19.9 & Broad em. $6600 \AA$ \\
\hline 328.276107 & 10.135809 & 20.0 & Broad em. $5450 \AA$ \\
\hline 338.817911 & 8.405663 & 20.3 & Broad em. $6850 \AA$ \\
\hline Variable & Stars & & \\
\hline 211.637100 & 20.845664 & 19.9 & Variable \\
\hline 241.766698 & 22.951423 & 19.8 & $\begin{array}{c}\text { Variable, period } \\
0.2278 \text { days. }\end{array}$ \\
\hline 250.411946 & 39.1151 & 20.2 & Variable \\
\hline 291.426967 & 38.535437 & 20.3 & Variable \\
\hline 341.865780 & 27.500455 & 20.5 & RRc? period 0.336 days \\
\hline 344.190408 & -5.472125 & 19.7 & $\begin{array}{l}\text { SX Phe period } \\
0.0402351 \text { days }\end{array}$ \\
\hline 355.394934 & 13.261791 & 19.8 & RRc period 0.423 days \\
\hline
\end{tabular}

Note.

a Objects that are not $a b$ type RR Lyr based on their Deimos spectra or on the period derived from their PTF light curves.

the distance of a candidate will be overestimated by $12 \%$ if it is actually a type $c$ rather than the more common type $a b$ RR Lyr. Furthermore, the $v_{r}-\phi$ relationship for $\mathrm{H} \alpha$ of the overtone pulsators may be different from that derived for the RR Lyr $a b$ that we are using.

Most importantly, type $c$ RR Lyr can be eliminated using a period-amplitude diagram, as is shown for the SDSS Stripe 82 sample in Figure 4. The type $c$ variables have smaller periods and smaller amplitudes of variation than do the fundamentalmode RR Lyr $a b$. There is essentially no overlap between them in this diagram.

\section{The Radial Dependence of the Velocity Dispersion}

Our ultimate goal is the determination of the mass of the MW out to as close to the virial radius as possible. We intend to use our sample of RR Lyr as massless point-source test particles. In support of this effort, we have ignored RR Lyr in the Sgr stream. However, there may be previously unknown structures whose stars may be moving with nonvirialized velocities. So before determining the velocity dispersion, we look for evidence from our data regarding the possible presence of new substructures. A search for previously unknown lowluminosity galactic satellites in the outer halo that have not yet been disrupted was conducted by Sesar et al. (2014) by using the RR Lyr from the PTF as indicators, but there was no detection, although the derived upper limit is high. With our $v_{r}$ survey we can look for evidence for the presence of more diffuse and more extended structures.

At the large distances we probe, the dispersion of the line-ofsight velocity, which is what we measure, is essentially identical to the dispersion of $v_{r}$, the radial velocity as seen from the Galactic center. We first consider the sample as a whole. Figure 8 shows a histogram of the entire sample of 112 RRab. We see a rather broad range spread between -220 and $+220 \mathrm{~km} \mathrm{~s}^{-1}$. This may be the result of a wide spread in $v_{r}$ at all galactocentric distances, of a trend with $r$, of the presence of outliers due to halo 


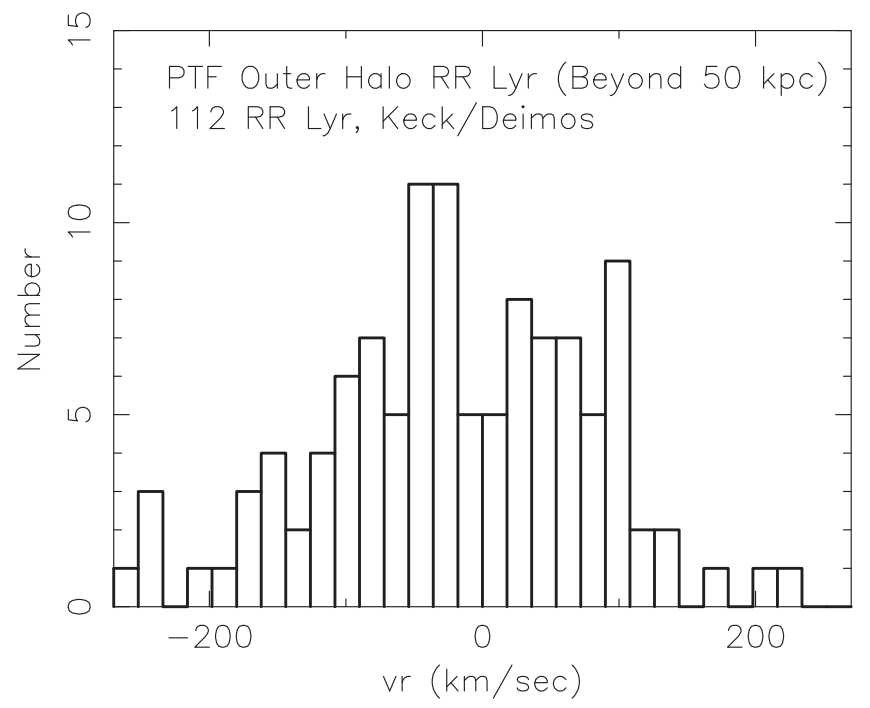

Figure 8. Histogram of the Keck/Deimos $v_{r}$ for the sample of 112 RR Lyr in the outer halo of the Milky Way beyond $50 \mathrm{kpc}$.

structures, or of contamination in the sample with objects that are not RRab. As discussed in Section 6.2, we believe that our sample has few such contaminants.

In an effort to identify whether outliers are present, we carried out an exercise where we began with the full sample, calculating the $v_{r}, \sigma\left(v_{r}\right)$, and the median distance. We then removed the largest outlier in $\mid v_{r}-\left\langle v_{r}\right\rangle$ (last)|, where "last" refers to the mean $v_{r}$ found in the previous iteration. We continued doing this repeatedly. The result for $v_{r}, \sigma\left(v_{r}\right)$, and the median distance is shown in Figure 9 for 24 such trials. First, we note that the median distance begins for the entire sample at $74.1 \mathrm{kpc}$ and slowly decreases, ending up after 24 deletions at $71.8 \mathrm{kpc}$. The $\sigma\left(v_{r}\right)$ for the entire sample treated as a whole begins at $98 \mathrm{~km} \mathrm{~s}^{-1}$ and ends up at $60 \mathrm{~km} \mathrm{~s}^{-1}$. It falls quite rapidly initially, suggesting the presence of some outliers, and then after about eight RRab are removed, the decline becomes more gradual. At the same time, the mean $v_{r}$ starts at about $-19 \mathrm{~km} \mathrm{~s}^{-1}$ and rises to $+3 \mathrm{~km} \mathrm{~s}^{-1}$ at the end of the 24 trials. So the halo has no, or at most very small, net motion.

To proceed further, we need to look into potential variations with distance and to whether we can find any more clues regarding the presence of outliers. The $v_{r}(\mathrm{GSR})$ we have determined for 112 candidate RR Lyr with distances beyond $50 \mathrm{kpc}$ in the MW halo are shown as a function of $r$ in Figure 10. In this figure, the stars are divided into three distance regimes, with the intermediate one being $70-85 \mathrm{kpc}$, and the most distant group, which contains 26 stars, ranges outward from 85 to $109 \mathrm{kpc}$, with four at distances exceeding $100 \mathrm{kpc}$. The first point to note is that the mean $v_{r}(\mathrm{GSR})$ for each of the three groups (shown as large stars in the figure) is close to $0 \mathrm{~km} \mathrm{~s}^{-1}$; the mean values and other statistics are given in Table 5. This is yet another indication that our values of $v_{r}(\mathrm{GSR})$ inferred from our $v_{r}$, as corrected for phase within the RR Lyr period, are in general valid.

Before computing the velocity dispersion, we need to decide whether there are genuine outliers and how to handle them. These are important as they may be a manifestation of previously unknown large-scale substructures in the halo. Figure 10 shows several outliers, and we have chosen $\left|v_{r}\right|<200 \mathrm{~km} \mathrm{~s}^{-1}$ as the cutoff for outliers for the sample within $85 \mathrm{kpc}$, dropping to $170 \mathrm{~km} \mathrm{~s}^{-1}$ outside that distance. The number of outliers in each

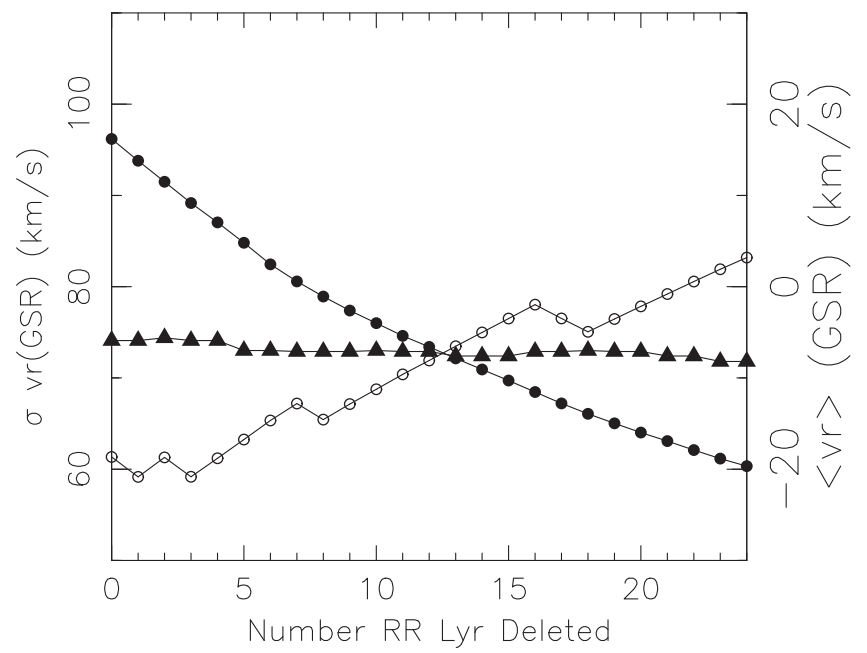

Figure 9. Tests sequentially deleting the largest outlier in $\left|\left(v_{r}-\left\langle v_{r}\right\rangle\right)\right|$, where the mean $v_{r}$ is that of the previous iteration, are shown as filled circles for $\sigma\left(v_{r}\right)$, as filled triangles for the median distance, and as open circles for the median distance of our RRab sample in the outer halo. The left axis gives the vertical scale for $\sigma\left(v_{r}\right)$, while the right axis gives the vertical scale for the other two curves.

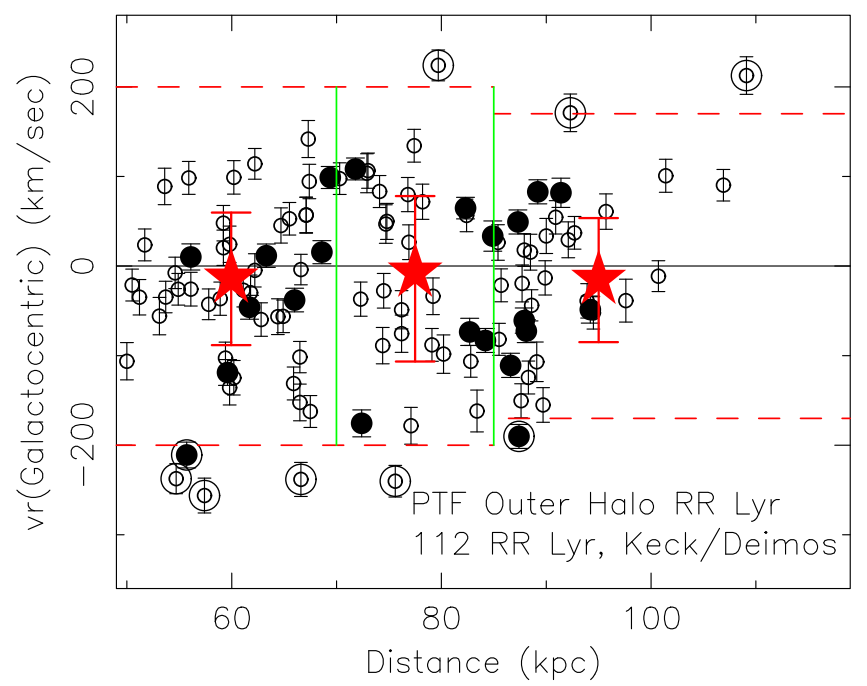

Figure 10. Radial velocities in the GSR are shown as a function of distances for our sample of 112 RR Lyr selected from the PTF with Keck/Deimos moderate-resolution spectra. Filled circles denote stars with two Deimos spectra; open circles have one spectrum. $1 \sigma$ error bars are shown for each RR Lyr. The regions (for both high and low $v_{r}$ ) considered outliers in $v_{r}$ are indicated by the dashed horizontal lines. The two vertical lines denote the boundaries between the close, middle, and far samples. The large stars are located at the median distance for each of these three samples in $X$, at the mean $v_{r}(\mathrm{GSR})$ in $Y$, and their error bars indicate the velocity dispersion for each of the three distance groups, ignoring the outliers. The nine outliers are circled. See Table 5 for detailed statistics of the $v_{r}(\mathrm{GSR})$ distribution.

distance range is given in Table 5. With this definition, there are a total of nine outliers from the $112 \mathrm{RRab}$ in our Keck $v_{r}$ sample. They are circled in Figure 10.

We first look at the low outliers. There are only five major low outliers. These five RR Lyr have $v_{r}<-200 \mathrm{~km} \mathrm{~s}^{-1}$ and distances between 51 and $73 \mathrm{kpc}$. All of them have excellent quality 1 light curves. Figure 11 shows the position on the sky of the $112 \mathrm{RR}$ Lyr candidates in our Keck/Deimos $v_{r}$ sample. These five low outliers (indicated as blue stars in the figure) are 


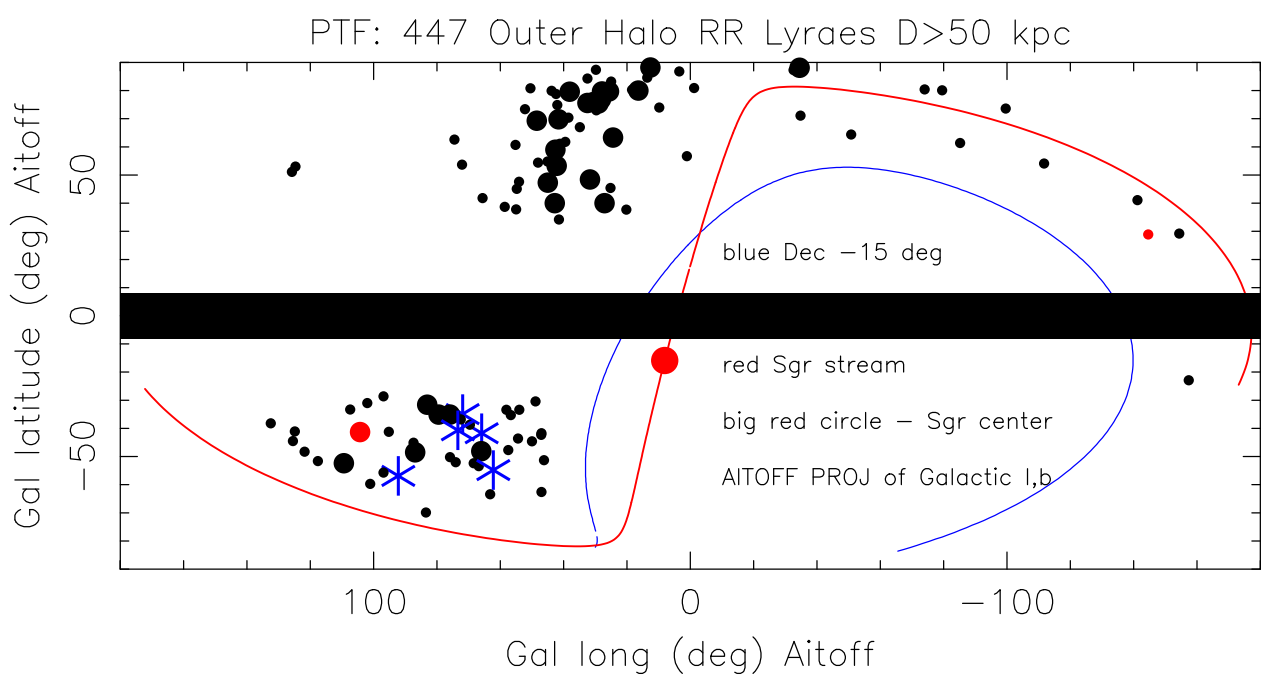

Figure 11. Location on the sky in galactic coordinates of the PTF outer halo RR Lyr stars with $v_{r}$ from Keck/Deimos in an Aitoff projection of Galactic coordinates. The larger dots denote RR Lyr with distances beyond $85 \mathrm{kpc}$. The five large blue stars indicate the only stars of the sample of 116 that have $v_{r}(\mathrm{GSR})<-200 \mathrm{~km} \mathrm{~s}{ }^{-1}$, while the red points have $v_{r}(\mathrm{GSR})>200 \mathrm{~km} \mathrm{~s}^{-1}$. The area around the Galactic plane that was excluded is indicated by the solid bar. The locus of the Sgr stream is denoted by the red curve, while the nucleus of the Sgr dwarf galaxy is indicated by the large red circle. Decl. $-15^{\circ}$ is indicated by the blue curve.

Table 5

Characteristics of the $v_{r}$ Distribution

\begin{tabular}{lccc}
\hline \hline$r$ Range & $N$ & $\begin{array}{c}<v_{r}(\mathrm{sys})>^{\mathrm{a}} \\
\left(\mathrm{km} \mathrm{s}^{-1}\right)\end{array}$ & $\begin{array}{c}\sigma^{\mathrm{b}} \\
\left(\mathrm{km} \mathrm{s}^{-1}\right)^{\mathrm{b}}\end{array}$ \\
\hline All & & & \\
$50<r<70$ & 51 & -27.4 & $97.4(95.3)$ \\
$70<r<85$ & 35 & -25.0 & $103.5(101.5)$ \\
$50<r<85$ & 86 & -26.2 & $99.4(97.4)$ \\
$85<r<106$ & 26 & 6.2 & $91.9(89.7)$ \\
& & & \\
Outliers ${ }^{\mathrm{c}}$ & & & $78.7(76.1)$ \\
$50<r<70$ & 47 & -9.3 & $89.4(87.1)$ \\
$70<r<85$ & 33 & -26.0 & $83.1(80.7)$ \\
$50<r<85$ & 80 & -16.2 & $68.0(65.0)$ \\
$85<r<109$ & 23 & -1.35 & \\
\hline
\end{tabular}

Notes.

${ }^{a}$ All velocities are in the GSR.

${ }^{\mathrm{b}}$ The $\sigma$ values are followed by values in parentheses that have a measurement uncertainty of $20 \mathrm{~km} \mathrm{~s}^{-1}$ removed in quadrature.

${ }^{\mathrm{c}}$ Outliers are defined as $\left|v_{r}\right|>170 \mathrm{~km} \mathrm{~s}^{-1}$ for $r>85 \mathrm{kpc},>200 \mathrm{~km} \mathrm{~s}^{-1}$ for $50<r<85 \mathrm{kpc}$.

confined to a small region on the sky with R.A. between $329^{\circ}$ and $10^{\circ}$ and decl. between $4^{\circ}$ and $15^{\circ}$. A blowup of this region on the sky is shown in Figure 12. The green points indicate RR Lyr in the Pisces overdensity, discovered by Sesar et al. (2007) as a linear stream at a distance of $\sim 80 \mathrm{kpc}$ within the SDSS stripe 82; however, recall that Stripe 82 is a narrow equatorial stripe only 1.27 wide extending from R.A. $20^{\mathrm{h}}$ to R.A. $4^{\mathrm{h}}$. A more recent discussion of this structure is given by Nie et al. (2015), but this structure is more distant than the set of five low outliers in our sample. An examination of Figures 11 and 12 combined with Figure 10 strongly suggests that these five stars belong to some previously unknown diffuse outer halo structure that extends over about $40^{\circ}$, perhaps from a disrupted satellite. These five low outliers are sufficiently far from the plane of the Sgr streams that it is unlikely that they are part of it. We therefore consider them as potentially not virialized. The

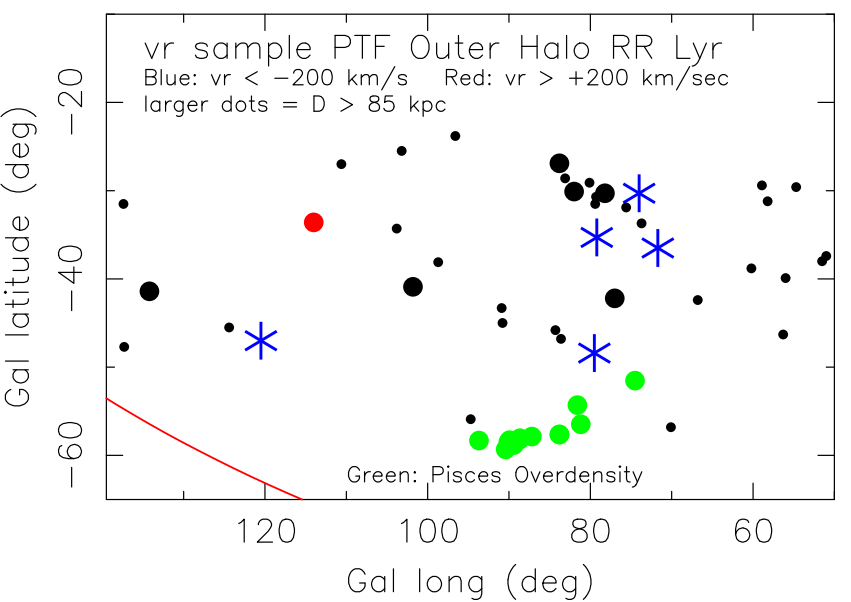

Figure 12. The $v_{r}$ sample shown in a plot of Galactic coordinates for a small subset of the total area on the sky covered. All the candidates with $v_{r}<-200 \mathrm{~km} \mathrm{~s}^{-1}$, indicated as large blue stars, lie within this small area on the sky. The location of the RR Lyr in the Pisces overdensity found by Sesar et al. (2007) is shown in green. The red curve denotes the Sgr stream.

choice of the $v_{r}$ cutoff adopted for RR Lyr with $r>85 \mathrm{kpc}$ of $\left|v_{r}\right|<170 \mathrm{~km} \mathrm{~s}^{-1}$ is an estimate based on Figure 10 .

Table 5 (see, also, Figure 13) gives the statistics of the sample of $112 \mathrm{RR}$ Lyr when divided into three distance ranges, and when only two groups are used, with a boundary at $85 \mathrm{kpc}$. Values for $\sigma\left[v_{r}(\mathrm{GSR})\right]$ are given as calculated from the measured $v_{r}(\mathrm{GSR})$ of each RR Lyr, and also with a $20 \mathrm{~km} \mathrm{~s}^{-1}$ measurement error removed. They are calculated for the full sample, and also for the case where the major outliers have been excluded. Since the removal of only a few outliers considerably reduces the velocity dispersion within each distance range, we consider our choice of cutoffs for outliers as reasonable. For example, for the outermost group of RR Lyr with $r>85 \mathrm{kpc}, \sigma\left[v_{r}(\mathrm{GSR})\right]$ is reduced from 90 to $65 \mathrm{~km} \mathrm{~s}^{-1}$ by removing only three outliers from our sample of $26 \mathrm{RR} \mathrm{Lyr}$ in this distance range.

Note that with the outliers eliminated, the velocity dispersion is quite low, not exceeding $87 \mathrm{~km} \mathrm{~s}^{-1}$ beyond $50 \mathrm{kpc}$, and for the outermost stars with $r>85 \mathrm{kpc}, \sigma\left[v_{r}(\mathrm{GSR})\right] \sim 65 \mathrm{~km} \mathrm{~s}^{-1}$. 


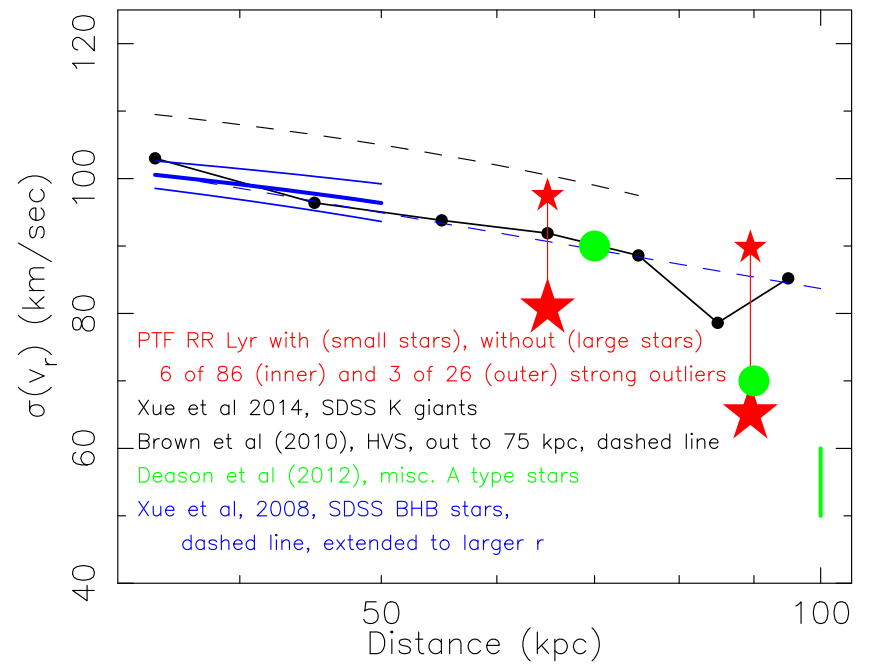

Figure 13. $\sigma\left(v_{r}\right)$ in the galactocentric rest frame shown for our inner and outer sample of RRab from the PTF (split at $85 \mathrm{kpc}$ ), with (large red stars) and without (smaller red stars) eliminating the strong outliers (three in the outer sample, and eight in the inner sample). Values that have been derived in several recent studies by Xue et al. (2008, whose extrapolation to larger $r$ is indicated by a dashed line), Xue et al. (2014), Brown et al. (2014), and Deason et al. (2012) are also indicated. Note that the $X$-axis has a logarithmic scale.

Clearly a larger sample of tracers with accurate distances and $v_{r}$ beyond $50 \mathrm{kpc}$ is desirable. We are working on it, but it will take several years to enlarge our sample of 112 RRab beyond $50 \mathrm{kpc}$ with measured $v_{r}$ by a substantial factor.

\section{The Radial Distribution of the PTF RR Lyr Sample}

With considerable caveats, we present the radial distribution in the outer halo of the MW for our sample of $447 \mathrm{RR}$ Lyr candidates. We assume an isotropic spherical halo. The major concern is the serious incompleteness in our sample of outer halo RR Lyr stars at the largest distances probed, i.e., beyond $90 \mathrm{kpc}$.

One might also worry about an increasing number of interlopers (i.e., not genuine RRab) in the sample as the distance increases and the light-curve quality decreases owing to increasing observational uncertainties in each individual observation and to a lower fraction of detections coupled with an increasing fraction of upper limits for a given number of epochs of observation. However, the small spread of the period distribution and the strong period-amplitude correlation shown in Figures 5 and 4, as well as the behavior of the quartiles of period and amplitude as a function of distance (see Figure 3), suggest that our sample is not contaminated by interlopers even at the largest distances we probe. Note that a careful examination of Figure 3 does support the suggestion that there is a strong increase in incompleteness of our sample of RR Lyr at the largest distances included in our sample.

We can assess the importance of incompleteness by considering the fraction of upper limits instead of detections among RRab stars that have many epochs of observation and that span the full range in distance probed here. Figure 14 shows some relevant data, specifically the fraction of upper limits among the available images in the PFS database for a sample 80 RR Lyr stars at the close end of our sample $(\sim 50 \mathrm{kpc})$ versus 50 of the most distant ones (i.e., beyond $95 \mathrm{kpc}$ ). As we had no control over the cadence or of the selection of fields to be observed on a given night at that time, the number of observations of a given field (at least at the time

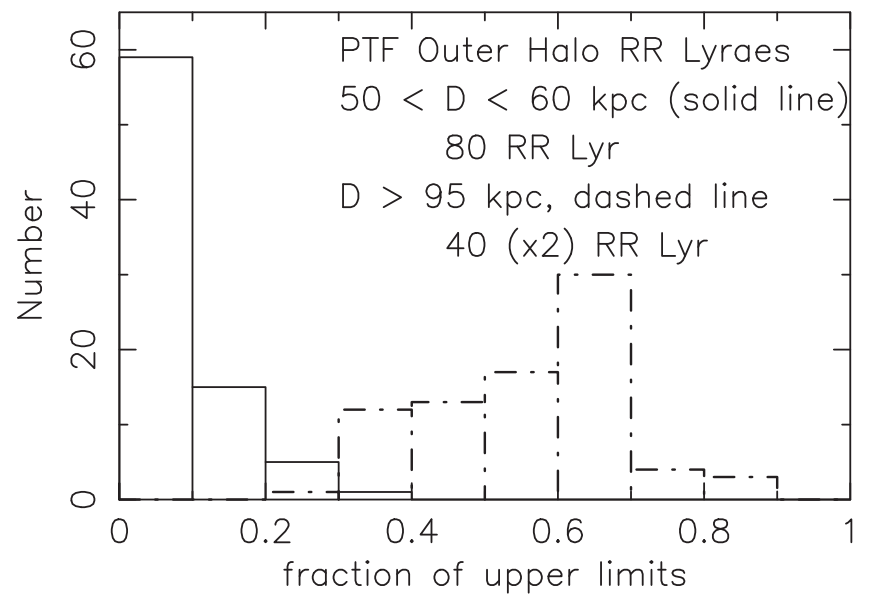

Figure 14. Fraction of upper limits among the total observed epochs of the PTF shown for samples of candidate RR Lyr with distances $\sim 55 \mathrm{kpc}$ vs. those with distances $>95 \mathrm{kpc}$. For the most distant RRab stars, a much larger fraction of the PTF images do not result in a detection of the candidate RR Lyr.

that the sample was constructed) depended on how many times a field was observed by other PTF projects.

For the nearer RR Lyr in our sample, the fraction of upper limits is low, usually less than $10 \%$, while for the most distant ones, the fraction of upper limits is typically $\sim 60 \%$. Our PTF RR Lyr sample was selected in 2014; hence, given the much smaller number of images of each field in the database at that time compared to the present values given in Table 2, a larger fraction of the most distant RR Lyr will not be picked up as candidate RR Lyr, as their light curves would not have contained more than 30 detections at that time. Although, as indicated earlier in Section 5, the ephemerides have been checked recently and updated as necessary, the list of candidates has not. Redoing the selection from the PTF seems unjustified given that a high-quality PS1 RR Lyr catalog with careful determination of its completeness and purity will be released shortly (Hernitschek et al. 2016; Sesar et al. 2017c). We can safely assume that there is an incompleteness of at least a factor of two for the most distant part of our sample; the actual completeness correction at the faint end of our PTF sample could be even larger.

With regard to the issue of contamination of the Keck $v_{r}$ sample by interlopers, we compare the number of candidate RR Lyr with $\operatorname{Pr}>0.8$ as a function of distance with the distribution in distance of our sample of $112 \mathrm{RR}$ Lyr with Keck/Deimos $v_{r}$. Note that 326 of the 447 candidates meet this probability restriction, while essentially all of candidates selected for Keck spectroscopy have $\operatorname{Pr}>0.8$. This ratio is given as a function of distance in Table 6. There are many candidates at the near end of the sample, but our goal was to get spectra of as many distant stars as possible, so candidates at the closer end of our sample were not observed unless no suitable distant candidate had a phase within the allowed range during that part of the night. As shown in Table 6, only $21 \%$ of the candidates with $r<64 \mathrm{kpc}$ have Keck $v_{r}$, while this fraction is $\sim 50 \%$ from 64 to $99 \mathrm{kpc}$, beyond which it drops to $\sim 38 \%$. If the sample of candidates was seriously contaminated with interlopers as the distance increased, this success fraction should have fallen significantly. We can therefore assume that the fraction of interlopers is not rising significantly toward the faint end of the sample, until a distance of at least $90 \mathrm{kpc}$, beyond which the sample is small. 
Table 6

Fraction of Candidate RR Lyr That Have Been Confirmed

\begin{tabular}{lccc}
\hline \hline$r$ Range & $\begin{array}{c}N(\text { RR Lyr) } \\
\text { Confirmed } \\
\text { RR Lyr } \\
\text { with } v_{r}{ }^{b}\end{array}$ & $\begin{array}{c}N(\text { RR Lyr) } \\
\operatorname{Pr}>0.8\end{array}$ & $\begin{array}{c}\text { Fraction }^{\mathrm{a}} \\
(\text { Confirmed/ } \\
\text { Candidates })\end{array}$ \\
\hline $50<r<64$ & 33 & 155 & 0.21 \\
$64<r<78$ & 38 & 86 & 0.44 \\
$78<r<85$ & 15 & 30 & 0.50 \\
$85<r<92$ & 14 & 25 & 0.56 \\
$92<r<99$ & 9 & 16 & 0.56 \\
$r>99$ & 3 & 8 & 0.38 \\
\hline
\end{tabular}

Notes.

a The ratio of the number of candidate RR Lyr selected from the PTF database in 2014 with $\operatorname{Pr}>0.8$ to those from this sample with Keck/Deimos $v_{r}$ for each distance bin

${ }^{b}$ The number of RR Lyr in this distance bin from the PTF sample that have been confirmed with Keck/Deimos spectroscopy and have a $v_{r}$ given in Table 2 .

Another way to approach the same issue is to examine the current PTF light curves for those RR Lyr candidates that are not in the Keck $v_{r}$ sample. If there is serious contamination that is dependent on distance by stars that are not RRab (presumably more contamination at larger distances), the fraction of these RR Lyr candidates that have quality 3 light curves (light curves that do not suggest that the star is an RR Lyr) will rise substantially with distance. We have carried out this check for candidates over a wide range in distance. For candidates at distances closer than $60 \mathrm{kpc}, 94 \%$ of them show high-quality light curves. This fraction falls to $85 \%$ for those between 65 and $70 \mathrm{kpc}$. It remains above $80 \%$ out to $95 \mathrm{kpc}$, beyond which it drops to $\sim 70 \%$. This change with distance of the potential fraction of contaminants is small enough that the effect on the power-law fit should not be large, at least within $95 \mathrm{kpc}$.

Figure 15 presents the number of candidates in bins in distance, with both axes of the plot using a logarithmic scale. Also shown are a number of power-law fits. The upwardpointing arrow indicates a correction for an incompleteness of $50 \%$ in the RR Lyr sample at the largest distances probed arising from the large fraction of upper limits in their PTF light curves. It is clear that a volume density law of $\rho(r) \propto r^{-4}$ is a reasonable fit from 50 to $85 \mathrm{kpc}$ and, with an incompleteness correction of a factor of two, would be a good fit out to $100 \mathrm{kpc}$.

\section{Comparison with Previous Results}

\subsection{Comparison with Other Samples of MW Outer Halo Stars}

As a result of many recent large stellar surveys, our knowledge of the outer halo of the MW is improving very rapidly. Outer halo stellar samples are increasing in size and distance range probed. For comparison, the early discussion of the kinematics of the halo by Kinman et al. (1996) used only a total of $67 \mathrm{RR}$ Lyr and BHB stars in the inner halo out to $r<15 \mathrm{kpc}$; they found a velocity dispersion of $\sim 110 \mathrm{~km} \mathrm{~s}^{-1}$. However, samples of outer halo stars with spectroscopic $v_{r}$, particularly in the crucial region beyond $r=80 \mathrm{kpc}$, such as ours are growing very slowly.

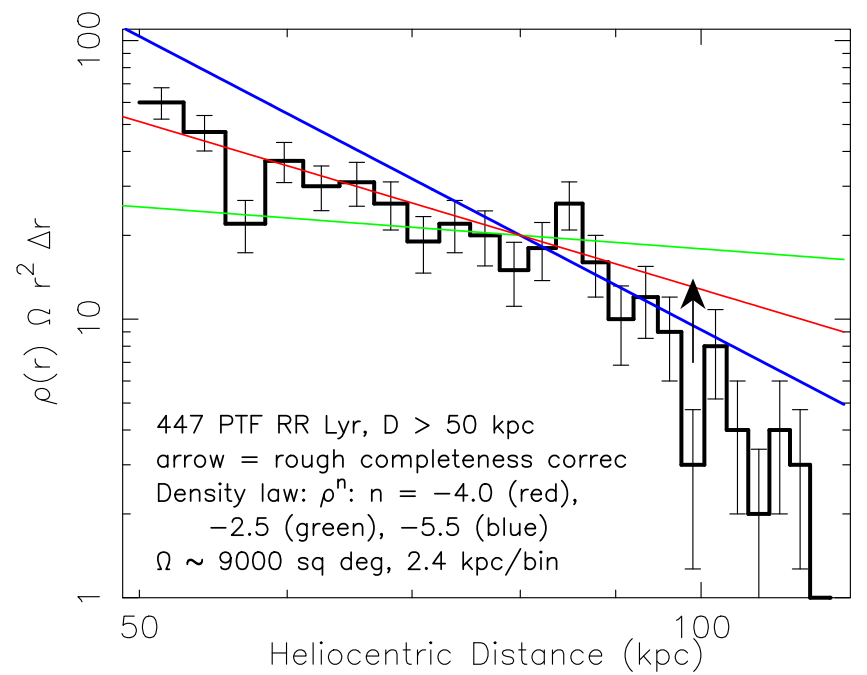

Figure 15. Histogram with distance of the 447 candidate RR Lyr stars from the PTF sample beyond $50 \mathrm{kpc}$. Power laws for $n=-2.5,-4.0$, and -5.5 are shown. The data are reasonably well fit for $n \sim-4$ out to $\sim 90 \mathrm{kpc}$, after which a steeper slope is seen. However, at that distance, the incompleteness effects are severe; a guess at the minimum completeness correction at such distances is shown by the upward-pointing arrow.

The CRTS (see, e.g., Mahabal et al. 2011) has been in operation since 2006, mining the data stream from three telescopes $(0.7 \mathrm{~m}, 1.0 \mathrm{~m}$, and $1.5 \mathrm{~m}$ in diameter $)$ in the mountains north of Tucson, Arizona, which are operated by the Lunar and Planetary Laboratory at the University of Arizona and whose primary mission is the detection of nearEarth asteroids. The photometric calibration and cadence of the CRTS are not as well controlled as those of the PTF, but the time span of the imaging and hence of the light curves is more than a decade. Drake et al. (2014) used this database to produce the Catalina Surveys Periodic Variable Star Catalog, which has roughly 16,800 RRab variables. The maximum distance of the RRab in their survey is $\sim 60 \mathrm{kpc}$; however, the bulk of their sample is closer than $40 \mathrm{kpc}$. Our sample begins at $50 \mathrm{kpc}$, and we have eliminated the Sgr stream, while the CRTS catalog has not. We find that the overlap between our sample of 447 RR Lyr and their sample is only 32 stars. The agreement of the derived period for the variables in common between the two surveys is less than 0.0010 days for 24 of the 32 stars in common, while the largest difference is 0.0016 days. The more recent southern extension of the Catalina Sky Surveys RR Lyr catalog, Torrealba et al. (2015), has no overlap in sky coverage with our Palomar-based survey.

Very recently Iorio et al. (2017), in a paper not yet accepted, have produced a catalog of RR Lyr by combining the first Gaia data release (Brown et al. 2016) with the Two Micron All Sky Survey (Skrutskie et al. 2006). Their sample has $~ 21,600 \mathrm{RR}$ Lyr and is confined to the inner halo. Thus, there is a very large overlap with the CRTS sample of Drake et al. (2014), but Iorio et al. (2017) only reach out to a heliocentric distance of $20 \mathrm{kpc}$, and thus there is no overlap with our sample, whose minimum distance is $50 \mathrm{kpc}$.

The huge database of SDSS, coupling uniformly measured multicolor photometry from its deep imaging over a large fraction of the northern sky and uniformly reduced spectra, was a breakthrough. It was used by Xue et al. (2008) to isolate a sample of $\sim 2400 \mathrm{BHB}$ stars with $v_{r}$ that reaches out to $r \sim 40 \mathrm{kpc}$, with very limited coverage out to $50 \mathrm{kpc}$; Xue 
et al. (2011) give a slightly improved selection of BHB stars from the same material. More recently, Xue et al. (2014) used the database of the Sloan Extension for Galactic Understanding and Exploration (SEGUE; Yanny et al. 2009) to select a sample of 6036 distant $\mathrm{K}$ giants. They developed probabilistic procedures to obtain their luminosities, claiming to have thus achieved a median accuracy of $16 \%$ in their distances. Their sample extends out to $\sim 80 \mathrm{kpc}$, although almost all of the stars beyond $60 \mathrm{kpc}$ are in the Sgr stream or other known halo substructures.

The K-giant sample of Xue et al. (2014), when cleaned of known substructures, primarily the Sgr stream, has 1757 stars with distances beyond $10 \mathrm{kpc}$, but in the outer halo it is significantly smaller than our sample, which begins at $50 \mathrm{kpc}$. The purged sample of Xue et al. (2014) has only two K giants beyond $65 \mathrm{kpc}$, with the most distant at about $75 \mathrm{kpc}$. Our sample reaches significantly farther out in the MW halo, with a median distance of $73 \mathrm{kpc}$. Furthermore, the distances to our RR Lyr sample are much more accurate than those of $\mathrm{K}$ giants.

The hypervelocity star survey (Brown et al. 2014) has carried out extensive spectroscopy of very blue stars in the outer halo selected from SDSS photometry. While SDSS colors are used, this is one of the few surveys besides ours that obtains their own spectra. The kinematics of the majority of their sample of the late B-type outer halo stars found in the course of this work are discussed in Brown et al. (2010). Their data set contains 910 late B and early A such stars, almost all of which are BHB stars with a small contamination of less luminous blue stragglers. The bulk of their sample is closer than $50 \mathrm{kpc}$.

Bochanski et al. (2014) selected a sample of 404 candidate very distant $M$ giants based on their near-IR colors from UKIDSS combined with optical colors from SDSS and undetectable proper motions (to rule out nearby $M$ dwarfs). Two of these were spectroscopically confirmed and appear to be extremely distant, with their estimated minimum distances being $130 \mathrm{kpc}$. Their sample of very distant $\mathrm{M}$ giants selected via photometry has roughly $80 \%$ contamination, which can only be resolved by spectroscopy; photometry alone is insufficient. Furthermore, $\mathrm{M}$ giants in the outer halo are a very biased indicator, as they can only arise from a metal-rich population and presumably are located in potentially nonvirialized, initially compact infalling structures, if in fact their distances and classifications are correct. As noted by Bochanski et al. (2014), these stars lie close to the Sgr plane. The recent model of the Sgr stream by Dierickx \& Loeb (2017) suggests that these $M$ giants are located within the Sgr stream; it successfully reproduces their distance and low $v$ (GSR). Sesar et al. (2017b) have recently identified some of these spurs in the Sgr stream at distances exceeding $100 \mathrm{kpc}$ using the PS1 RR Lyr sample. However, given the high contamination fraction of their $\mathrm{M}$ giant sample, the amount of observing time that would be required to generate a clean large sample of such distant $M$ giants is prohibitive, and furthermore a sample of $\mathrm{M}$ giants would not probe the bulk of the outer halo of the MW.

Slater et al. (2017) isolate a sample of $~ 4000$ distant giants in the halo with wide-field imaging using a narrow bandwidth filter covering the region of the $\mathrm{Mg}$ triplet at $5170 \AA$, which is well known to be a good giant/dwarf discriminator. This is combined with broadband SDSS imaging. Extensive statistical treatment using population synthesis modeling is required to clean the sample of numerous dwarf interlopers, and the distances of individual objects are quite uncertain. The sample of $\sim 4000$ giants reaches out to $80 \mathrm{kpc}$.

Deason et al. (2012) attempted to build up a sample of more distant $(D>80 \mathrm{kpc})$ BHB stars by stacking multiepoch photometry from Stripe 82 of the SDSS (and other regions with multiple images) to isolate candidate BHB stars, but these are too faint to have SDSS spectroscopy. They obtained lowresolution $(\lambda / \Delta \lambda \sim 800)$ spectra using FORS2 on the Very Large Telescope to try to separate BHB stars from contamination by brighter blue stragglers, which outnumber by a factor of more than four the desired BHB stars. The final sample has only seven faint BHB stars. Deason et al. (2012) then add a small number of other potential outer halo stars with highly uncertain distances, as well as the dwarf satellites of the MW.

The only sample that reaches out to the distances probed by our RR Lyr sample with a substantial number of stars beyond $50 \mathrm{kpc}$ is that of Deason et al. (2014), which uses BHB and blue straggler stars from the SDSS DR9. There are several issues that afflict this sample, particularly contamination with blue stragglers and, more seriously, with QSOs. Extensive color modeling, taking into account photometric scattering, was used to try to remove contaminants, which outnumber the desired BHB stars by a large factor.

It is clear that the sample of distant RR Lyr from the PTF with Keck radial velocities presented here has unique characteristics. It is a clean sample with few interlopers, and each star has a highly accurate distance. At the present time, and even after the release in 2017 November of the PS1 sample by Sesar et al. (2017c), ours is the only reliable sample with at least a modest number of tracer stars beyond $80 \mathrm{kpc}$ in the outer halo with measured $v_{r}$.

\subsection{The Density Profile in the Outer Halo}

The determination of the density profile in the outer halo of a large set of massless tracer stars is clearly a crucial input to determining the mass of the MW. Given the limited data, the solution is usually expressed as a power-law fit to the density versus distance. Our preliminary result based on a large sample of RR Lyr variables is given in Section 8 and is shown in Figure 15. We find that a power law in $r$ with a slope of $\sim-4$ is consistent with the stellar density $\rho(r)$ derived from the distances of our RR Lyr sample. This assumes an isotropic spherical halo. With larger samples one can also solve for the flattening profile of the halo, but we could not attempt this. Bovy et al. (2016), based on an analysis of the Pal 5 and GD-1 stellar streams, suggest that the axis ratio of the dark matter's halo density distribution is 1.05 within the inner $20 \mathrm{kpc}$, providing some support to our assumptions, although Iorio et al. (2017) suggest that the inner halo has a substantial oblateness that decreases at larger Galactic radii. There seems to be a general consensus that the outer halo is less oblate than the inner halo.

Our result contradicts that of Deason et al. (2014), who claim that beyond $50 \mathrm{kpc}$ there is a striking drop in the stellar halo density. Although in their earlier paper (Deason et al. 2011) they found a power-law fit of $\alpha=-4.6$ for the region $27<r<40 \mathrm{kpc}$ (the maximum $r$ reached), Deason et al. (2014) find a power-law slope of -6 beyond $50 \mathrm{kpc}$, with even steeper slopes (power-law index -6 to -10 ) favored at larger radii. On the other hand, De Propris et al. (2010), who used a sample of $666 \mathrm{BHB}$ stars from the $2 \mathrm{dF}$ quasar redshift survey, found a very shallow slope for the density in the outer halo of 
$-2.5 \pm 0.2$ and a velocity dispersion that increases with $r$, reaching a huge $\sigma\left(v_{r}\right)$ exceeding $200 \mathrm{~km} \mathrm{~s}^{-1}$ at $r \sim 80 \mathrm{kpc}$ over the two lines of sight probed. Our data do not support the results of either of these two studies. Our sample is much cleaner with much better distances than the samples of either of these two analyses.

A number of other analyses have been published recently that agree with our halo density distribution to within the uncertainties. Among the many samples of outer halo stars discussed above in Section 9.1, the large SDSS/SEGUE samples of $\mathrm{K}$ giants stand out for their size and spatial coverage. The latest analysis of such is that of Xue et al. (2016). As the luminosity of K giants depends strongly on the metallicity, they had to use forward modeling techniques to fit the spatial distribution and abundance distribution simultaneously. They found that a power-law slope with index $-3.8 \pm 0.1$ is a good fit to the number density profile of the halo beyond $r \sim 20 \mathrm{kpc}$. Das \& Binney (2016) reanalyze this sample using an extended distribution function to find that the density distribution power-law index is -4 at large radii out to $80 \mathrm{kpc}$. Kafle et al. (2014) combine the BHB and K-giant samples from the SDSS/SEGUE to find a slope of -4.5 in the halo beyond $\sim 20 \mathrm{kpc}$. The recent work by Slater et al. (2017) using SDSS photometry coupled with imaging in a narrowband filter centered at the $\mathrm{Mg}$ triplet to eliminate dwarfs also targets $\mathrm{K}$ giants. They use sophisticated color-magnitude diagram modeling and population synthesis to derive a halo density profile $\rho \propto r^{-3.5}$ from 30 to $80 \mathrm{kpc}$.

Thus, as discussed above, there seems to be a growing consensus that in the outer halo of the MW, at least out to $85 \mathrm{kpc}$, the stellar density can be represented as a power law with a slope of -3.5 to -4 . This is quite close to the slope found in the inner halo, at least from $20 \mathrm{kpc}$ outward, by several groups (see, e.g., Xue et al. 2016).

\subsection{The Velocity Dispersion in the Outer Halo}

The behavior of the $v_{r}$ of a sample of massless tracers as a function of distance provides important clues as to the potential and total mass of the MW. Toward this goal, several of the studies referenced in Section 9.1 have measured $v_{r}$ for a large fraction of the members of their sample. In particular, those based on SDSS and its successors (i.e., SEGUE) fall into this class. In this section we compare our derived $\sigma\left(v_{r}\right)$ as a function of distance for our RRab sample (shown in Table 5 and in Figure 13) with those of other groups.

The two large samples of outer halo stars based on the SDSS and SEGUE, i.e., the BHB sample of Xue et al. (2008) (see also Xue et al. 2011) and the K-giant sample of Xue et al. (2016), both of which reach out to $r \sim 50 \mathrm{kpc}$, have been analyzed by many different groups using various sophisticated modeling techniques to derive properties of the outer halo. The latest result from these samples is Xue et al. (2016), where references to earlier work can be found.

Xue et al. $(2008,2011)$ derived the radial trend of $\sigma\left(v_{r}\right)$ out to $50 \mathrm{kpc}$, where they found $\sigma\left(v_{r}\right) \sim 95 \mathrm{~km} \mathrm{~s}^{-1}$. The spatial range of this relation was extended by Deason et al. (2012), who added a small number of more distant objects. Kafle et al. (2014), who derived their own sample of K giants from the SEGUE data, also found a similar value of $\sigma\left(v_{r}\right)$ of $\sim 100 \mathrm{~km} \mathrm{~s}^{-1}$ for $r \sim 50 \mathrm{kpc}$; see their Figure 1 .

Figure 13 illustrates some of these results from the literature compared to our relationship for $\sigma\left(v_{r}\right)$ as a function of $r$ between 50 and $100 \mathrm{kpc}$. With the exception of De Propris et al. (2010), all of these investigations, including ours presented here, are in reasonable agreement regarding the velocity dispersion of the outer halo stars as a function of distance from 50 to $100 \mathrm{kpc}$ within the regime probed by each group, $50-100 \mathrm{kpc}$ in our case. All recent studies find $\sigma\left(v_{r}\right) \sim 90 \mathrm{~km} \mathrm{~s}^{-1}$ at $50 \mathrm{kpc}$, dropping lower as $r$ increases. The hypervelocity star survey (Brown et al. 2014) derives the same general decline of $\sigma\left(v_{r}\right)$ with $r$ but has $\sigma\left(v_{r}\right)$ roughly $20 \mathrm{~km} \mathrm{~s}^{-1}$ higher at all $r$ probed than our result and that of most recent work.

The agreement on the spatial distribution $n(r)$ among the various studies, among the most recent of which is Xue et al. (2016), is also satisfactory out to perhaps $60 \mathrm{kpc}$; from $\sim 30$ to $\sim 60 \mathrm{kpc}$ all groups agree that the number density of tracers can be represented by a power law with index of about -4 . There are only two surveys beyond that, our work and that of Deason et al. (2014), and there is a major disagreement at these larger distances between us, with Deason et al. (2012) claiming a very rapid drop in the number density beyond $50 \mathrm{kpc}$. They find a power law of -6 with distance beyond $50 \mathrm{kpc}$, dropping to slopes of -6 to -10 at larger distances. Unless we have badly underestimated our contamination problems, which at least in the sample selected for Keck spectroscopy is highly unlikely given the quality ratings of the light curves and the period - phase relation for our sample shown in Figure 4, we advocate that our results are more reliable, given the substantial contamination of the Deason et al. (2012) sample by QSOs, for which the corrections they use may not be adequate.

We thus conclude that the outer halo at $r>70 \mathrm{kpc}$ is cold, and its radial velocity dispersion is low. These factors suggest, in accordance with several recent analyses, a low total mass for the MW. For our RRab survey based on the PTF database, the key issues are the purity and completeness of the sample and the potential impact of substructure, which we suggest may produce the outliers in $v_{r}$ in these distant outer halo samples of "massless and virialized" tracer stars. The new Pan-STARRS RR Lyr catalog by Hernitschek et al. (2016) and by Sesar et al. $(2017 \mathrm{c})$ will allow future investigations to avoid most if not all of these concerns.

\section{Summary}

RR Lyr stars of type $a b$ are ideal massless tracers that can be used to study the outer halo of the MW. Because they have (to first order) a fixed luminosity, their periods are about 0.5 days, they are common in old metal-poor stellar populations, and their amplitude of variation is substantial, reaching up to $\sim 1 \mathrm{mag}$, they are easily found in any multicolor imaging survey with extensive temporal coverage. Since they are blue, even when they are in the outer halo of the MW, they stand out against the numerous redder foreground stars and can be distinguished from quasars by the nature of their variability, quasars being nonperiodic variables, while the other blue halo stars (BHBs and blue stragglers) can be eliminated as being nonvariable. RR Lyr are thus ideal probes of the outer halo that can be found at great distances in the current generation of large stellar surveys and whose distances can be measured to high accuracy with just a light curve.

We present here a sample of 112 RRab beyond $50 \mathrm{kpc}$ in the outer halo of the MW for which we have obtained moderateresolution spectra with Deimos on the Keck II Telescope. Four of these have distances exceeding $100 \mathrm{kpc}$. These were selected 
from a much larger set of 447 candidate RR Lyr that were datamined using machine-learning techniques applied to the light curves of variable stars in the PTF database. The observed radial velocities taken at the phase of the variable corresponding to the time of observation were converted to systemic radial velocities in the GSR. This only works well when the ephemerides of the variable stars are accurately known.

From our sample of 112 RR Lyr with Keck $v_{r}$ we determine the radial velocity dispersion in the outer halo of the MW to be $\sim 90 \mathrm{~km} \mathrm{~s}^{-1}$ at $50 \mathrm{kpc}$, falling to about $65 \mathrm{~km} \mathrm{~s}^{-1}$ near $100 \mathrm{kpc}$ once a small number of major outliers are removed. The five very low $v_{r}$ (GSR) stars, all of which have $v_{r}(\mathrm{GSR})<-200 \mathrm{~km} \mathrm{~s}^{-1}$, are surprisingly close together on the sky at a distance of about $60 \mathrm{kpc}$, but there is no known structure at that distance in that part of the sky.

With reasonable estimates of the completeness of our sample of 447 candidates and assuming a spherical halo, we find that the stellar density in the outer halo declines as $r^{-4}$. Most, but not all, other recent works corroborate this functional form.

The problems we have faced have been in the accuracy of the ephemerides for the RR Lyr sample and in issues of completeness and non-RR Lyr interlopers. Further exploration of the issue of substructure in the outer halo requires a larger sample. The new Pan-STARRS RR Lyr catalog by Hernitschek et al. (2016) and by Sesar et al. (2017c) provides this and will allow investigations that we expect to carry out in the near future to avoid most if not all of these concerns. Ultimately LSST will allow techniques similar to those we used to identify RR Lyr at even larger distances of up to several hundred kiloparsecs. Of course, spectroscopic follow-up of the very distant RRab we expect to find with LSST will require the next generation of extremely large telescopes beyond the current $10 \mathrm{~m}$ Kecks.

We are grateful to the many people who have worked to make the Keck Telescope and its instruments a reality and to operate and maintain the Keck Observatory. The authors wish to extend special thanks to those of Hawaiian ancestry on whose sacred mountain we are privileged to be guests. Without their generous hospitality, none of the observations presented herein would have been possible.

We thank the referee for helpful detailed comments that improved this paper.

This work uses data obtained with the $1.2 \mathrm{~m}$ Samuel Oschin Telescope at Palomar Observatory as part of the Palomar Transient Factory project, a scientific collaboration among the California Institute of Technology, Columbia University, Las Cumbres Observatory, the Lawrence Berkeley National Laboratory, the National Energy Research Scientific Computing Center, the University of Oxford, and the Weizmann Institute of Science; and the Intermediate Palomar Transient Factory project, a scientific collaboration among the California Institute of Technology, Los Alamos National Laboratory, the University of Wisconsin, Milwaukee, the Oskar Klein Center, the Weizmann Institute of Science, the TANGO Program of the University System of Taiwan, and the Kavli Institute for the Physics and Mathematics of the Universe.

The Intermediate Palomar Transient Factory project is a scientific collaboration among the California Institute of Technology, Los Alamos National Laboratory, the University of Wisconsin, Milwaukee, the Oskar Klein Center, the Weizmann Institute of Science, the TANGO Program of the
University System of Taiwan, and the Kavli Institute for the Physics and Mathematics of the Universe.

The PTF database (DR3) is now publicly available at https://www.ptf.caltech.edu/news/DR3. It includes photometry through 2015 January 28.

Funding for SDSS-III has been provided by the Alfred P. Sloan Foundation, the Participating Institutions, the National Science Foundation, and the U.S. Department of Energy Office of Science. The SDSS-III website is http://www.sdss3.org/.

SDSS-III is managed by the Astrophysical Research Consortium for the Participating Institutions of the SDSS-III Collaboration, including the University of Arizona, the Brazilian Participation Group, Brookhaven National Laboratory, Carnegie Mellon University, University of Florida, the French Participation Group, the German Participation Group, Harvard University, the Instituto de Astrofisica de Canarias, the Michigan State/Notre Dame/JINA Participation Group, Johns Hopkins University, Lawrence Berkeley National Laboratory, Max Planck Institute for Astrophysics, Max Planck Institute for Extraterrestrial Physics, New Mexico State University, New York University, Ohio State University, Pennsylvania State University, University of Portsmouth, Princeton University, the Spanish Participation Group, University of Tokyo, University of Utah, Vanderbilt University, University of Virginia, University of Washington, and Yale University.

This research has made use of the NASA/IPAC Extragalactic Database (NED), which is operated by the Jet Propulsion Laboratory, California Institute of Technology, under contract with the National Aeronautics and Space Administration.

J.G.C. and B.S. acknowledge NSF grant AST-0908139 to J.G.C. for partial support during the initial early stages of this project. S.R.B. and K.H. thank the Caltech Summer Undergraduate Research Fellowship (SURF) program for support.

\section{ORCID iDs}

Judith G. Cohen (iD https://orcid.org/0000-0002-8039-4673 Branimir Sesar (ib https://orcid.org/0000-0002-0834-3978 Shrinivas R. Kulkarni (D) https://orcid.org/0000-00015390-8563

Thomas A. Prince (iD https://orcid.org/0000-0002-8850-3627 Eric Bellm (1) https://orcid.org/0000-0001-8018-5348

\section{References}

Ahn, C. P., Alexandroff, R., Allende Prieto, C., et al. 2014, ApJS, 211, A17 Baade, W. 1926, AN, 228, 359

Belokurov, V., Koposov, S. E., Evans, N. W., et al. 2014, MNRAS, 437, 116 Benedict, G. F., McArthur, B. E., Feast, M. W., et al. 2011, AJ, 142, 187 Bochanski, J. J., Willman, B., Caldwell, N., et al. 2014, ApJL, 790, L5 Bovy, J., Bahmanyar, A., Fritz, T. K., \& Kalilvayalil, N. 2016, ApJ, 833, 31 Braga, V. F., Dall'Ora, M., Bono, G., et al. 2015, ApJ, 799, A165 Breiman, L. 2001, Mach. Learn., 45, 5

Brown, W. R., Geller, M. J., \& Kenyon, S. J. 2014, ApJ, 787, A89 Brown, W. R., Geller, M. J., Kenyon, S. J., \& Diaferio, A. 2010, AJ, 139, 59 Caceres, C., \& Catelan, M. 2008, ApJS, 179, 242

Carrasco, D., Barrientos, L. F., Pichara, K., et al. 2014, A\&A, 584, A44 Chaboyer, B. 1999, in Post-Hipparcos Cosmic Candles, ed. A. Heck \& F. Caputo (Dordrecht: Kluwer), 111

Chadid, M., Sneden, C., \& Preston, G. W. 2017, ApJ, 835, 187

Cohen, J. G. 1992, ApJ, 400, 528

Cohen, J. G., \& Huang, W. 2009, ApJ, 701, 1053

Cohen, J. G., \& Huang, W. 2010, ApJ, 719, 931

Das, P., \& Binney, J. 2016, MNRAS, 460, 1725

Deason, A. J., Belokurov, V., \& Evans, N. W. 2011, MNRAS, 416, 2903

Deason, A. J., Belokurov, V., Evans, N. W., et al. 2012, MNRAS, 425, 2840 
Deason, A. J., Belokurov, V., Koposov, S. E., \& Rockosi, C. 2014, ApJ, 787, 30

De Propris, R., Harrison, C. D., \& Mares, P. J. 2010, ApJ, 719, 1582

Dierickx, M. I., \& Loeb, A. 2017, ApJ, 836, A92

Drake, A. J., Graham, M. J., Djorgovski, S. G., et al. 2014, ApJS, 213, 9

Faber, S., Phillips, A. C., Kibrick, R. I., et al. 2003, Proc. SPIE, 4841, 1657

Fazio, G., et al. 2004, ApJS, 154, 10

Brown, A. G. A., Vallenari, A., Prusti, T., et al. 2016, A\&A, 23, A2

Grillmair, C. J., \& Carlin, J. L. 2016, in Tidal Streams in the Local Group and Beyond, Astrophysics \& Space Science Library, ed. H. Newberg \& J. Carlin (Berlin: Springer), 87

Grillmair, C. J., Laher, R., Surace, J., et al. 2010, in ASP Conf. 434, Astronomical Data Analysis Software and Systems XIX, ed. Y. Mizumoto, K.-I. Morita, \& M. Ohishi (San Francisco, CA: ASP), 28

Hernitschek, N., Schlafly, E. F., Sesar, B., et al. 2016, ApJ, 817, A73

Hodapp, K. W., Kuhn, J., Thornton, R., et al. 2004, in Scientific Detectors for Astronomy, Astrophysics and Space Science Library Vol. 300, ed. P. Amico, J. W. Beletic, \& J. E. Beletic (Berlin: Springer), 501

Honeycutt, R. K. 1992, PASP, 104, 435

Iorio, G., Belokurov, V., Erkal, D., et al. 2017, MNRAS, submitted (arXiv:1707.03833)

Kafle, P. R., Sharma, S., Lewis, G. F., \& Bland-Hawthorn, J. 2014, ApJ, 794, A59

Keller, S. C., Murphy, S., Prior, S., Da Costa, G., \& Schmidt, B. 2008, ApJ, 678,851

Kinman, T. D., Pier, J. R., Sunzeff, N. B., et al. 1996, AJ, 111, 1164

Kirby, E. N., Lanfranchi, A., Simon, J. D., et al. 2011, ApJ, 727, 78

Klein, C. R., Richards, J. R., Butler, N. R., \& Bloom, J. S. 2014, MNRAS, 440, L96

Laher, R. R., Surace, J., Grillmair, C. J., et al. 2014, PASP, 126, 674

Law, N. M., Kulkarni, S. R., Dekany, R. G., et al. 2009, PASP, 121, 1395

Levitan, D., Fulton, B. J., Groot, P. J., et al. 2011, ApJ, 739, 68

Longmore, A. J., Fernley, J. A., \& Jameson, R. F. 1986, MNRAS, 220, 279

Longmore, A. J., Fernley, J. A., \& Jameson, R. F. 1990, MNRAS, 247, 684

Madore, B. F., Hoffman, D., Freedman, W. L., et al. 2013, ApJ, 776, A135

Mahabal, A., Djorgovski, S. G., Drake, A. J., et al. 2011, BASI, 39, 387

Marconi, M., Coppola, G., Bono, G., et al. 2015, ApJ, 808, A50

McCauliff, S., Jenkins, J. M., Catanzarite, J., et al. 2015, ApJ, 806, 6

Miceli, A., Rest, A., Stubbs, C. W., et al. 2008, ApJ, 678, 865

Michalik, D., Lindegren, L., \& Hobbs, D. 2015, A\&A, 574, A115

Nie, J. D., Smith, M. C., Belokurov, V., et al. 2015, ApJ, 810, A153

Nikutta, R., Hunt-Walker, N., Nenkova, M., Ivezić, Z., \& Elitzur, M. 2014 MNRAS, 442, 3361
Nun, I., Pichara, K., Protopapas, P., \& Kim, D.-W. 2014, ApJ, 793, A23

Ofek, E. O., Frail, D. A., Breslauer, B., et al. 2011, ApJ, 740, 65

Ofek, E. O., Laher, R., Law, N., et al. 2012, PASP, 124, 62

Rahmer, G., Smith, R., Velur, V, et al. 2008, Proc. SPIE, 7014, 163

Rau, A., Kulkarni, S. R., Law, N. M., et al. 2009, PASP, 121, 1334

Richards, J. W., Starr, D. L., Butler, N. R., et al. 2011, ApJ, 733, 10

Schlafly, E. F., \& Finkbeiner, D. P. 2011, ApJ, 373, A103

Schlafly, E. F., Green, G., Finkbeiner, D. F., et al. 2014, ApJ, 789, A15

Schlegel, D., Finkbeiner, D. P., \& Davis, M. 1998, ApJ, 500, 525

Schorck, T., Christlieb, N., Cohen, J. G., et al. 2009, A\&A, 507, 817

Sesar, B. 2012, AJ, 144, 114

Sesar, B., Banholzer, S. R., Cohen, J. G., et al. 2014, ApJ, 793, A135

Sesar, B., Cohen, J. G., Levitan, D., et al. 2012, ApJ, 755, 134

Sesar, B., Fouesneau, M., Price-Whelan, A., et al. 2017a, ApJ, 838, A107

Sesar, B., Grillmair, C. J., Cohen, J. G., et al. 2013a, ApJ, 776, 26

Sesar, B., Hernitschek, N., Dierickx, M. I. P., Fardal, M. A., \& Rix, H. W. 2017b, ApJL, 844, L4

Sesar, B., Hernitschek, N., Ivezic, Z., et al. 2017c, AJ, 153, 204

Sesar, B., Ivezic, A., Lupton, R. H., et al. 2007, AJ, 134, 2236

Sesar, B., Ivezić, Z., Grammer, S. H., et al. 2010, ApJ, 708, 717

Sesar, B., Ivezić, Z., Sutart, J., et al. 2013b, AJ, 146, 21

Sesar, B., Jurić, M., \& Ivezić, Z. 2011, ApJ, 731, A4

Sharma, M., Nayak, J., Koul, M. K., Bose, S., \& Mitra, A. 2014, RAA, 14,1491

Skrutskie, M. F., Cutri, R. M., Stiening, R., et al. 2006, AJ, 131, 1163

Slater, C. T., Nidever, D. L., Munn, J. M., Bell, E. F., \& Majewski, S. R. 2017, ApJ, 832, A206

Soszynski, I., Udalski, A., Szymanski, M., et al. 2016, AcA, 66, 131

Stetson, P. B. 1996, PASP, 108, 851

Tonry, J. L., Stubbs, C. W., Lykke, K. R., et al. 2012, ApJ, 750, A99

Torrealba, G., Catelan, M., Drake, A. J., et al. 2015, MNRAS, 446, 2251

Vivas, A. K., Zinn, R., Andrews, P., Bailyn, C., et al. 2001, ApJL, 554, L33

Werner, M. W., Roellig, T. L., Low, F. J., et al. 2004, ApJS, 154, 1

Wesselink, A. J. 1969, MNRAS, 144, 297

Wetterer, C. J., \& McGraw, J. T. 1996, AJ, 112, 1046

Wright, E. L., et al. 2010, AJ, 140, 1868

Xue, X. X., Ma, Z., Rix, H.-W., et al. 2014, ApJ, 784, 170

Xue, X. X., Rix, H. W., Ma, Z., et al. 2016, ApJ, 809, A144

Xue, X. X., Rix, H. W., Yanny, B., et al. 2011, ApJ, 738, 79

Xue, X. X., Rix, H.-W., Zhao, G., et al. 2008, ApJ, 684, 1143

Yanny, B., Rockosi, C., Newberg, H. J., et al. 2009, AJ, 137, 4377

York, D. G., Adelman, J., Anderson, J. E., Jr., et al. 2000, AJ, 120, 1579

Zacharias, N., Finch, C., Girard, T., et al. 2010, AJ, 139, 2184 